\title{
IC 1590, A YOUNG CLUSTER EMBEDDED IN THE NEBULOSITY OF NGC 281
}

\author{
HARRY H. GUETTER ${ }^{1}$ \\ U.S. Naval Observatory, Flagstaff Station, P.O. Box 1149, Flagstaff, Arizona 86002 \\ Electronic mail: guetter@nofs.navy.mil \\ DAVID G. TURNER ${ }^{1,2}$ \\ Department of Astronomy and Physics, Saint Mary's University, Halifax. Nova Scotia, B3H 3C3, Canada \\ Electronic mail: turner@hercules.stmarys.ca \\ Received 1996 November 15; revised 1997 February 27
}

\begin{abstract}
Photoelectric and CCD photometry (to a limit of $V=17.0$ ) is presented for 61 and 243 stars, respectively-a total of 279 individual stars-in and about IC 1590, a young galactic cluster embedded in the nebulosity of NGC 281, the H II region S184. New spectroscopic observations are also presented for 20 stars in or near the cluster. A small amount of differential reddening is observed across the face of IC 1590, and is described by a visual reddening relation of slope $\mathrm{E}_{U-B} / \mathrm{E}_{B-V}=0.735$. From near infrared photometry of bright cluster members in the $I$ and $K$ bands and a variable-extinction analysis of ZAMS members, the value of $R=A_{V} / \mathrm{E}_{B-V}$, the ratio of total to selective absorption, is found to be $3.44 \pm 0.07$, slightly larger than what has been found for other nearby galactic fields. Most of the cluster reddening appears to originate in dust clouds which are $\sim 0.66 \mathrm{kpc}$ and $2.0-2.2 \mathrm{kpc}$ distant, the latter cloud lying in the immediate foreground of the cluster, which has a derived distance of $2.94 \pm 0.15 \mathrm{kpc}\left(V_{0}-M_{V}=12.34 \pm 0.11\right.$ s.d., \pm 0.03 s.e.). IC 1590 is extremely young and has an estimated age for its 63 identified probable members of $3.5( \pm 0.2) \times 10^{6}$ years, with very little evidence for age spread. Its well-defined sequence of 22 gravitationally-contracting stars, the choice of which is supported by star counts and reddening information, provides evidence for the extreme youth of the cluster's central trapezium system, HD 5005. According to the luminosity distribution of likely cluster members, the initial mass function for IC 1590 has a slope of $\Gamma=-1.00 \pm 0.21$, similar to what has been found for other very young clusters and associations. Given that the photometric study terminates at a magnitude limit marginally brighter than where embedded protostars and T Tauri variables belonging to the cluster are expected to be found, IC 1590 would make an excellent target for Hubble Space Telescope observations. (C) 1997 American Astronomical Society. [S0004-6256(97)01206-5]
\end{abstract}

\section{INTRODUCTION}

IC $1590\left(\mathrm{OCl} 313, \mathrm{C} 0049+563, \alpha_{1950}=00^{\mathrm{h}} \quad 49^{\mathrm{m}} \cdot 9\right.$, $\left.\delta_{1950}=+56^{\circ} 21^{\prime}, l=123^{\circ} 1, \quad b=-06^{\circ} 2\right)$ is an early-type galactic cluster which contains a group of stars clustered about the O-type trapezium system ADS 719 or HD 5005 (Sharpless 1954; Abt 1986). The cluster is embedded in the nebulosity of NGC 281, also identified as the bright $\mathrm{H} \mathrm{II}$ emission region Sharpless 184 of diameter $20 \mathrm{arcmin}$, and is relatively unusual among bright, young, northern hemisphere clusters in that most of the literature on it relates to studies at microwave and radio wavelengths. Prior to our present investigation the region had been omitted from programs of observation at visual wavelengths, an exception being a study by Henning et al. (1994) that included Strömgren sys-

\footnotetext{
${ }^{1}$ Visiting Astronomer, Kitt Peak National Observatory, National Optical Astronomy Observatories, which is operated by the Association of Universities for Research in Astronomy, Inc. (AURA) under co-operative agreement with the National Science Foundation.

${ }^{2}$ Guest Investigator, Dominion Astrophysical Observatory, Herzberg Institute of Astrophysics, National Research Council of Canada.
}

tem photometry for the brightest cluster stars.

Most of our knowledge of IC 1590 is inferred from studies outside of the optical region. The H II region NGC 281 is surrounded by an extensive $\mathrm{H}$ I cloud which is roughly twice as large, and several dark Bok globules are visible against its face. The ionized hydrogen seems to be associated with two $\mathrm{CO}$ molecular clouds which have been mapped in ${ }^{12} \mathrm{CO}$ and ${ }^{13}$ CO by Elmegreen \& Lada (1978), Leisawitz et al. (1988), and Henning et al. (1994). One of the clouds is located in the southwest quadrant of the emission nebula, coincident with a curtain of heavy obscuring dust. It is referred to in the literature as molecular cloud $\mathrm{N} 281 \mathrm{~B}$, or radio peak $\mathrm{A}$, and has a recessional velocity in the local standard of rest (LSR) system of almost $+44 \mathrm{~km} \mathrm{~s}^{-1}$. It contains an $\mathrm{H}_{2} \mathrm{O}$ maser source (Elmegreen \& Lada 1978), IRAS sources, and bipolar outflows (Henning et al. 1994), all of which are indicative of active star formation. The other cloud, N281A or radio peak $\mathrm{B}$, is located in the southeastern quadrant of the nebulosity and has an LSR velocity of $-30.5 \mathrm{~km} \mathrm{~s}^{-1}$, very similar to that of the $\mathrm{H}$ II region itself $\left(-26.5 \mathrm{~km} \mathrm{~s}^{-1}\right.$, Leisawitz 1988).

The radio source has been the subject of many other radio, millimeter, and infrared investigations (see Israel 1977 
for those completed prior to 1974; Henning et al. 1994). Leisawitz et al. (1988) derived a total molecular gas content for NGC 281 of $\sim 2 \times 10^{4} \mathscr{M b}_{\odot}$, while Roger \& Pedlar (1981), from synthesis interferometer measurements both in the continuum and at a wavelength of $21-\mathrm{cm}$, estimated the amount of H I and H II to be $10^{4} \mathscr{L}_{\odot}$ and $2.2 \times 10^{3} \mathscr{L b}_{\odot}$, respectively. They concluded that the ultraviolet flux needed to ionize the $\mathrm{H}$ II region could be supplied by the visible $\mathrm{O}$-type stars, and found that the emission region lies on the edge of an extensive H I cloud. Radial velocity measures of the molecular, atomic and ionized gas components suggest that the molecular clouds produce the ionized gas which flows towards the O-type stars, and that most of the $\mathrm{HI}$ is kinematically and spatially coincident with the CO clouds. Detailed schematic models for this particular star formation region have been presented by Roger \& Pedlar (1981) and by Johnson et al. (1981).

As is typically the case, the $\mathrm{H} \mathrm{I}$ and $\mathrm{H}$ II mass estimates are geometry dependent, and rely quite heavily on a rather uncertain distance estimate for the cluster- $2.0 \mathrm{kpc}$ to 2.2 $\mathrm{kpc}$ in older studies, but $3.5 \mathrm{kpc}$ according to Henning et al. (1994). That and the former neglect of the cluster by optical astronomers were the primary reasons why we decided to complete a more detailed study of the field. Initially that consisted mostly of $U B V$ photometry, but the program evolved into a more thorough study involving infrared photometry and faint star spectroscopy after we discovered that anomalous extinction might be present (Guetter et al. 1985). The reddening law appropriate for the cluster has a direct influence on such parameters as the reddening and distance of member stars, so it is important to study such features in detail in order to establish the cluster distance as accurately as possible. Since the earliest star in the cluster has a spectral type of either O5.5f (Conti \& Alschuler 1971) or $06.5 \mathrm{~V}((\mathrm{f})$ ) (Walborn 1973) and an $\mathrm{H}_{2} \mathrm{O}$ maser and IRAS sources are part of the complex, the cluster is clearly very young, with star formation still occurring in the molecular cloud southwest of it. As we demonstrate here, IC 1590 is an excellent example of a very young cluster in which pre-main-sequence stars can be found in abundance. It is therefore an ideal case for comparing theoretical predictions of pre-main-sequence evolution with observational data of high quality.

\section{PHOTOMETRIC AND SPECTROSCOPIC OBSERVATIONS}

Photoelectric measurements of stars in and around IC 1590 were initiated by Turner at Kitt Peak National Observatory (KPNO) using the $0.4 \mathrm{~m}$ reflector during one night in 1978 August and 3 nights in 1979 August, and were continued using the $0.9 \mathrm{~m}$ telescope during 3 nights in $1981 \mathrm{Sep}-$ tember. The $2.1 \mathrm{~m}$ telescope was also used in 1979 September to obtain widened photographic spectra at a dispersion of $103 \AA \mathrm{mm}^{-1}$ for four members of the trapezium system HD 5005. Additional photoelectric photometry was obtained by Guetter during 1980, 1984, and 1985 with a single-channel photometer attached to the $1.0 \mathrm{~m}$ Ritchey-Chretien reflector at the Flagstaff Station of the U.S. Naval Observatory. The 1980 filter set and detector are described by Guetter (1979), while those employed during the latter two observing periods
TABLE 1. Photoelectric standards

\begin{tabular}{|c|c|c|c|c|c|}
\hline Star & $V$ & $B-V$ & $U-B$ & $n$ & Remarks \\
\hline A & 9.87 & +0.53 & +0.02 & 3 & $\mathrm{BD}+55^{\circ} 175$ \\
\hline $\mathrm{B}$ & 8.08 & +0.65 & +0.23 & 3 & $\mathrm{BD}+55^{\circ} 178, \mathrm{HD} 4868, \mathrm{G} 0$ \\
\hline $\mathrm{C}$ & 9.25 & +0.05 & -0.33 & 3 & $\mathrm{BD}+55^{\circ} 179, \mathrm{~B} 9 \mathrm{III}(\mathrm{a})$ \\
\hline $\mathrm{D}$ & 9.38 & +1.02 & +0.67 & 3 & $\mathrm{BD}+55^{\circ} 182$ \\
\hline $\mathrm{E}$ & 9.74 & +0.50 & +0.01 & 3 & $\mathrm{BD}+55^{\circ} 184$ \\
\hline $\mathrm{F}$ & 9.59 & +0.44 & +0.00 & 3 & $\mathrm{BD}+55^{\circ} 187, \mathrm{~F} 0(\mathrm{HDE})$ \\
\hline $\mathrm{G}$ & 11.84 & +0.19 & +0.07 & 3 & \\
\hline $\mathrm{H}$ & 11.33 & +1.53 & +1.68 & 5 & \\
\hline I & 10.12 & +0.42 & +0.21 & 3 & \\
\hline $\mathbf{J}$ & 12.52 & +0.13 & -0.33 & 3 & \\
\hline $\mathrm{K}$ & 11.92 & +0.30 & +0.24 & 4 & \\
\hline $\mathrm{HD} 5005 \mathrm{abc}$ & 7.93 & +0.10 & -0.85 & 2 & \\
\hline HD 5005ab & 8.34 & +0.08 & -0.86 & $*$ & O6.5 V((f)) (b, brighter star) \\
\hline HD $5005 c$ & $9.19::$ & $+0.13::$ & $-0.82::$ & 4 & O8 $\operatorname{Vn}(b, c)$ \\
\hline HD 5005d & 9.78 & +0.06 & -0.86 & 8 & O9 Vn $(c, d)$ \\
\hline 52 & 13.08 & +1.20 & +1.06 & 4 & \\
\hline 54 & $13.42::$ & +0.17 & $-0.34::$ & 6 & Variable, double $\Delta \mathrm{m}=4 \mathrm{~m} 2$ \\
\hline 55 & 14.32: & +0.69 & $+0.10:$ & 3 & \\
\hline 56 & 14.36 & +0.27 & -0.09 & 3 & \\
\hline 65 & 10.78 & +0.09 & -0.66 & 12 & $\mathrm{~B} 1.5 \mathrm{Vn}(\mathrm{c})$ \\
\hline 68 & 14.05 & +0.20 & -0.14 & 13 & \\
\hline 70 & 14.63: & +0.86 & +0.22 & 10 & \\
\hline 92 & 10.42 & +0.06 & -0.71 & 3 & Double, $\Delta \mathrm{m}=3^{\mathrm{m}} \cdot \mathrm{B} 1 \mathrm{~V}(\mathrm{c})$ \\
\hline 93 & 12.40 & +0.16 & -0.46 & 4 & B3 V (c) \\
\hline 95 & 12.60 & +0.20 & -0.22 & 3 & B6 V (c) \\
\hline 149 & 13.91 & $+0.41:$ & $+0.17:$ & 6 & Sharpless h \\
\hline 150 & $13.67::$ & +0.25 & -0.19 & 7 & Sharpless j, B8 Vnne (c) \\
\hline 151 & $13.83::$ & +0.30 & -0.17 & 9 & Sharpless k, B8 Vnne (c) \\
\hline 152 & 12.55 & +0.19 & -0.47 & 11 & Sharpless e, B2.5 Vnn (c) \\
\hline 155 & 14.52 & +0.98 & +0.73 & 2 & Sharpless i \\
\hline 161 & 11.35 & +0.16 & -0.58 & 3 & $\mathrm{~B} 2 \mathrm{Vn}(\mathrm{c})$ \\
\hline 191 & 13.49 & +0.49 & $+0.41:$ & 7 & \\
\hline 194 & 11.65 & +0.35 & -0.47 & 3 & $\mathrm{~B} 1.5 \mathrm{Vn}(\mathrm{c})$ \\
\hline 195 & 10.87 & +0.18 & -0.58 & 11 & $\mathrm{~B} 1.5 \mathrm{~V}(\mathrm{c})$ \\
\hline 199 & 14.83 & +0.35 & -0.02 & 2 & \\
\hline 213 & 12.12 & +0.17 & -0.43 & 12 & B3 Vn (c) \\
\hline 214 & 11.26: & +0.15 & -0.58 & 13 & B2.5 Vne (c) \\
\hline 215 & 13.45: & +0.39 & -0.20 & 14 & $\mathrm{~B} 8 \mathrm{Ve}(\mathrm{c})$ \\
\hline 216 & 12.46 & +0.41 & +0.29 & 11 & \\
\hline 218 & 13.89 & +0.28 & +0.00 & 11 & \\
\hline 220 & 13.54 & +0.66 & +0.49 & 12 & \\
\hline 221 & 11.88 & +0.44 & +0.24 & 11 & \\
\hline 237 & 11.46 & +0.26 & +0.20 & 3 & \\
\hline
\end{tabular}

Notes: (a) Spectral type from Guetter.

(b) Spectral type from Walborn (1973).

(c) Spectral type from Turner.

(d) Spectral type from Abt (1986).

* Value deduced from measures for abc and c.

are identical to those used by Guetter \& Hewitt (1984).

The averaged photoelectric measurements are listed in Table 1, and bright stars in the field, denoted by upper case letters, are identified in Fig. 1. The remaining numbered objects are identified in Fig. 2, while the notation for the components of the trapezium system is that of Sharpless (1954). Colons appended to the data denote a mean scatter of between \pm 0.02 and \pm 0.04 , and double colons refer to deviations larger than \pm 0 m.04. The photometric accuracy is low for objects near HD 5005; it illuminates the emission nebulosity of NGC 281, within which the faint companions are embedded. In this region of patchy nebulosity the various sky measures depend very critically on where the aperture is 


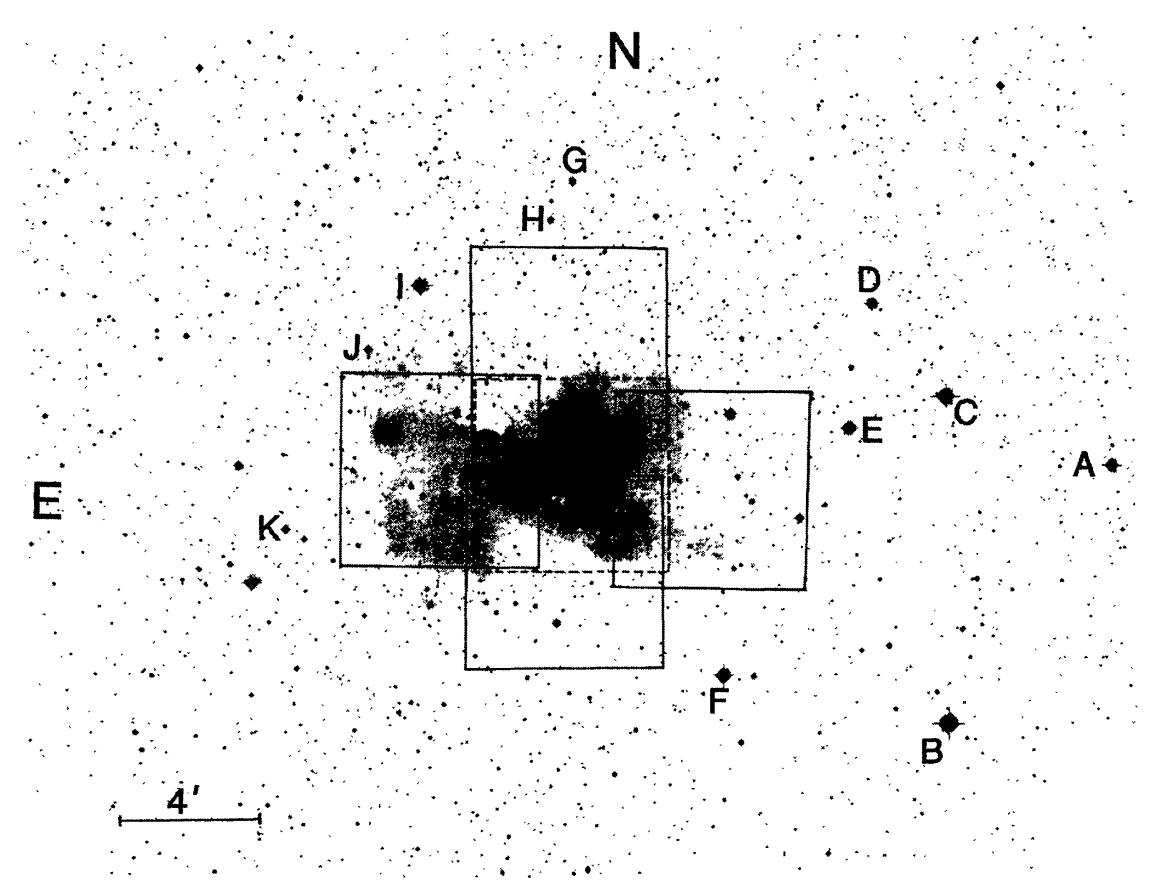

FIG. 1. A reproduction of a 3.4 hour exposure on a IIaO plate with GG13 filter taken with the $1.5 \mathrm{~m}$ reflector of the U.S. Naval Observatory showing the nebulosity of NGC 281 (Sharpless 184) in which the young galactic cluster IC 1590 is embedded. The five CCD fields are indicated by solid and dashed contours, and the letters refer to the stars listed in Table 1.

placed, and hence the sky corrections are difficult to establish reliably.

Between 1989 and 1992, four to five sets of CCD frames in each of the $V, B$, and $U$ bandpasses were obtained in five cluster fields located on the north, east, south, and west sides of $\mathrm{HD} 5005$, as well as centered on the trapezium system itself. The boundaries of the five CCD fields are shown schematically in Fig. 1; the four directional regions are delineated by solid lines, and dashed lines outline the central area. Observations were obtained using a $800 \times 800 \mathrm{TI}$ CCD detector mounted on the $1.0 \mathrm{~m}$ Ritchey-Chretien reflector with filters chosen to match the Johnson $U B V$ system. The detectortelescope combination provides a total field of view of $5.7 \times 5.7$ for square pixels of projected dimension 0."43. Each set of observations consisted of frames with integration times of 30-sec and 2-min, 1-min and 4-min, and 6-min and 24$\min$ in the $V, B$, and $U$ bandpasses, respectively, except for the central field where the exposures were halved. The observations were reduced using the DAOPHOT profile-fitting routine NSTAR (Stetson 1987), and the resulting magnitudes and colors were transformed to the Johnson $U B V$ system using the photoelectric standards listed in Table 1.

A comparison of CCD photometric results for $V$ frames of different exposure times indicated that, owing to saturation problems for bright stars on the long exposures and poor photon statistics for faint stars on the short exposures, stars with $V \leqslant 15.7$ on the short exposure images had the same photometric accuracy as objects with $13.4<V<17.2$ measured on the longer exposure images. All other standards were ignored in the image calibrations. Similarly, the limiting magnitude for short exposure $B$ images was found to be $B \leqslant 16.6$, while the range for comparable accuracy on long exposure images was $14.0<B<18.1$. Finally, in the same manner the limiting magnitude for short exposure $U$ images was established to be $U \leqslant 17.1$, while the range for comparable accuracy on long exposure images was $14.1<U<18.5$.

The calibrated CCD photometry is listed in Table 2, where the identifications are according to Fig. 2. The $\mathrm{X}$ and $\mathrm{Y}$ coordinates refer to the calculated distances in arcminutes from HD 5005 (in the positive sense for increasing right ascension and declination), and remarks are given at the end of the table. Three stars were too bright for the CCD survey, and their photoelectric data are listed in parentheses in the table. Two standards (54 and 155) were found to be variable, two objects (150 and 151) have poorly determined $V$ magnitudes, and one star (70) has a discordant $U-B$ color. For the remaining 20 standards, the CCD values of $V, B-V$, and $U-B$ are tied to their photoelectric equivalents with standard deviations of $\pm 0^{\mathrm{m}} \cdot 008, \pm 0^{\mathrm{m}} \cdot 005$, and $\pm 0^{\mathrm{m}} \cdot 007$, respectively. The $U$ magnitudes for faint stars near HD 5005 could not be measured accurately owing to the short exposures as well as the high sky background produced by the UV radiation of the O-type trapezium system. However, since DAOPHOT computes the sky corrections in patchy regions of nebulosity using an annulus about each star rather than from offset apertures, the CCD observations should be more accurate than their photoelectric counterparts. After the rejection of 12 stars that are clearly variable, the final average standard errors were found to be $\pm 0.008 \quad(n=235)$, \pm 0 m.008 $(n=235)$ and $\pm 0^{\mathrm{m}} \cdot 014(n=229)$ for $V, B-V$, and $U-B$, respectively, and each star was observed on an average of five different nights. Colons and double colons for the 


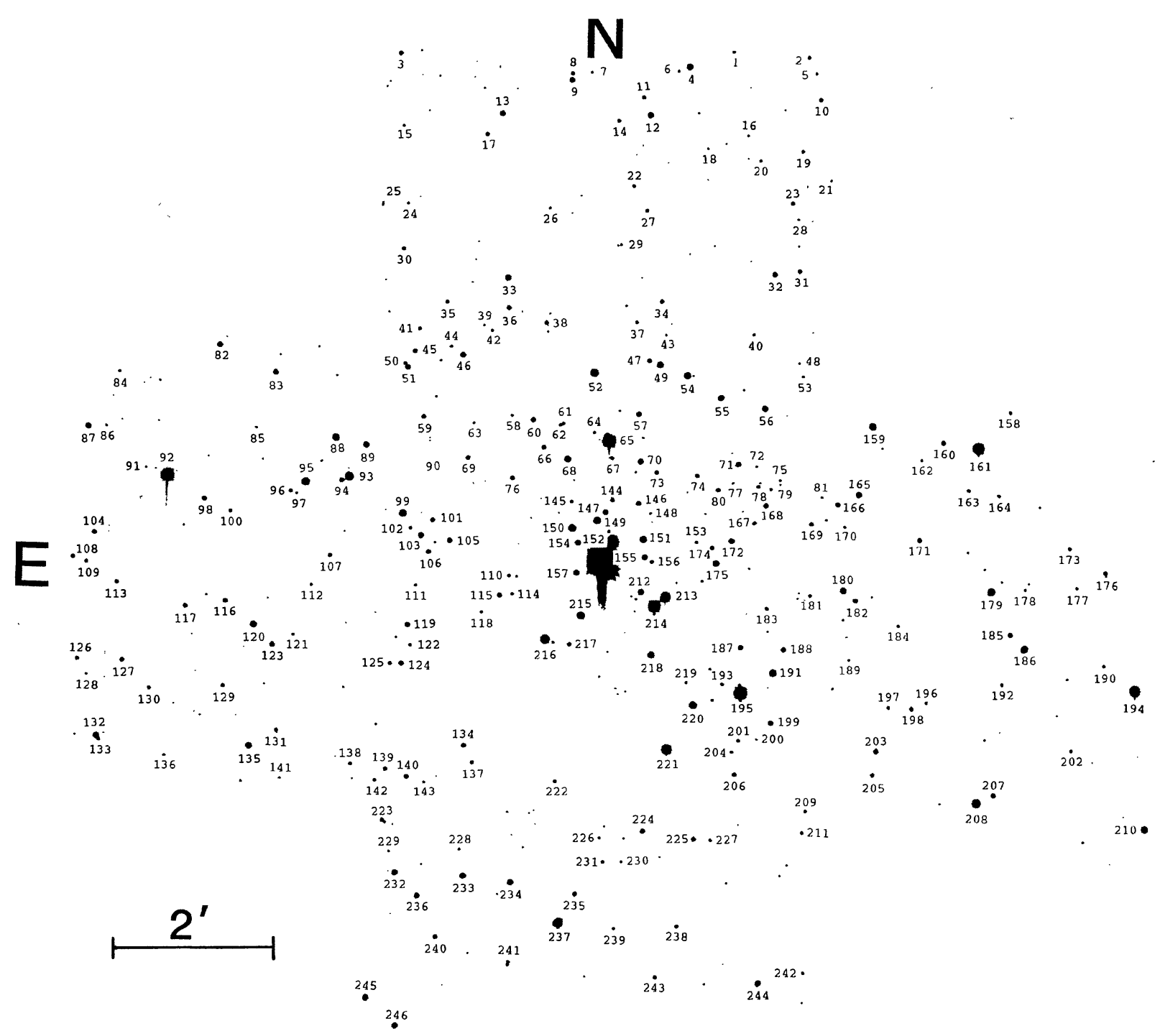

FIG. 2. A mosaic of the five CCD fields centered on HD 5005. The numbering system refers to the designations used in Tables 1 and 2.

Table 2 data have the same meanings as applied to the photoelectric measures.

For reference purposes, $U B V$ photoelectric measures were obtained during 1984 October for 19 additional field stars located within 0.5 of $\mathrm{HD} 5005$ using the equipment described previously. The results are listed in Table 3. Half of the stars have $\mathrm{MK}$ or $\mathrm{HD}(\mathrm{E})$ spectral classifications, and color classes can be estimated for the remainder of the sample. Classification spectra for three of the stars were obtained with the Sharpless spectrograph (Guetter 1968) attached to the $1 \mathrm{~m}$ reflector at the U.S. Naval Observatory.

CCD spectroscopic observations were also obtained for 14 stars in IC 1590 during the autumn of 1995 using the Cassegrain spectrograph on the $1.85 \mathrm{~m}$ Plaskett telescope of the Dominion Astrophysical Observatory. The spectra, which are at a dispersion of $60 \AA \mathrm{mm}^{-1}$, were recorded with the Observatory's SITe-1 detector, which has a wavelength resolution of $1.44 \AA$ at that dispersion. The reduced spectral images, which are shown in Fig. 3, were classified relative to a grid of similar spectra for MK standards and other earlytype stars that were observed for other purposes. The classifications resulting from both the photographic and CCD spectra are listed in the last column of Table 1.

\section{COLOR EXCESSES AND THE EXTINCTION LAW}

A rather extensive spectroscopic survey of stars in IC 1590 was considered essential for addressing questions about the nature of the extinction law in the cluster. A comparison of the observed $U B V$ colors for the 16 cluster stars of established MK type with the colors expected for such stars (see Turner 1989) yields the color excesses $\mathrm{E}_{U-B}$ and $\mathrm{E}_{B-V}$ plotted as filled symbols in Fig. 4. By way of comparison, similar color excesses were calculated for $21 \mathrm{OB}$ stars lying in the general vicinity of the cluster (plotted as open circles in Fig. 4), where the $U B V$ data and MK types are from Hiltner (1956), Morgan et al. (1955), and Table 3. 
TABLE 2. CCD photometry.

\begin{tabular}{|c|c|c|c|c|c|c|c|c|c|c|c|}
\hline Star & $V$ & $B-V$ & $U-B$ & $X$ & $Y$ & Star & $V$ & $B-V$ & $U-B$ & $X$ & $Y$ \\
\hline $\begin{array}{ll}\text { の゙ } \\
\text { |ே1 }\end{array}$ & 16.13 & $0.87:$ & 0.32 & -1.44 & 6.36 & 65 & $(10.78$ & 0.09 & $-0.66)$ & 0.03 & 1.47 \\
\hline$=2$ & 15.73 & 0.63 & 0.18 & -2.38 & 6.32 & 66 & 15.59 & 1.59 & 1.68 & 0.84 & 1.38 \\
\hline 3 & 15.04 & 0.84 & 0.30 & 2.72 & 6.26 & 67 & 16.39 & 0.77 & 0.12 : & -0.01 & 1.26 \\
\hline 4 & 13.54 & 0.77 & 0.29 & -0.90 & 6.17 & 68 & 13.99 & 0.20 & -0.17 & 0.55 & 1.24 \\
\hline 5 & 16.86 & 0.97 & 0.51 & -2.48 & 6.12 & 69 & 15.71 & 1.28 & 1.13: & 1.79 & 1.23 \\
\hline 6 & 16.55 & 0.81 & 0.21 & -0.77 & 6.11 & 70 & 14.65 & 0.89 & $0.50::$ & -0.37 & 1.23 \\
\hline 7 & 17.23 & 0.96 & 0.56 & 0.33 & 6.08 & 71 & 15.03 & 0.35 & 0.25 & -1.60 & 1.21 \\
\hline 8 & 15.68 & 0.90 & 0.41 & 0.57 & 6.06 & 72 & 17.21 & 0.83 & 0.25 & -1.82 & 1.19 \\
\hline 9 & 14.33 & 0.62 & 0.16 & 0.58 & 5.98 & 73 & 15.86 & 1.18 & 0.69 & -0.58 & 1.09 \\
\hline 10 & 15.32 & 1.29 & 0.87 & -2.54 & 5.79 & 74 & 15.79 & 0.73 & $0.14:$ & -1.09 & 1.06 \\
\hline 11 & 15.77 & 0.87 & 0.35 & -0.33 & 5.77 & 75 & 17.06 & 0.77 & 0.23 & -2.12 & 1.02 \\
\hline 12 & 13.76 & 0.59 & 0.26 & -0.42 & 5.55 & 76 & 15.41 & 1.24 & 0.81 & 1.23 & 0.98 \\
\hline 13 & 14.09 & 1.16 & 0.76 & 1.44 & 5.54 & 77 & 17.28 & 0.81 & 0.25 & -1.54 & 0.97 \\
\hline 14 & 16.05 & 1.00 & 0.52 & -0.02 & 5.48 & 79 & 16.55 & 1.23 & 0.77 & -2.01 & 0.90 \\
\hline 78 & 16.14 & 1.06 & 0.55 & -1.86 & 0.93 & 80 & 15.70 & 0.96 & 0.52 & -1.36 & 0.88 \\
\hline 15 & 17.04 & 1.48 & $\ldots$ & 2.66 & 5.36 & 81 & 17.09 & 1.21 & $\ldots$ & -2.65 & 0.81 \\
\hline 16 & 16.94 & 0.86 & 0.33 & -1.64 & 5.32 & 82 & 14.38 & 0.70 & 0.15 & 4.83 & 2.60 \\
\hline 17 & 15.40 & 1.05 & 0.72 & 1.62 & 5.27 & 83 & 14.38 & 0.67 & 0.21 & 4.12 & 2.27 \\
\hline 18 & 17.26 & 0.74 & 0.18 & -1.15 & 5.15 & 84 & 16.74 & 1.12 & $0.78:$ & 6.07 & 2.24 \\
\hline 19 & 15.94 & 0.95 & 0.51 & -2.33 & 5.14 & 85 & 17.06 & 0.73 & 0.18 & 4.35 & 1.56 \\
\hline 20 & 16.79 & 1.37 & $\ldots$ & -1.80 & 5.01 & 86 & 17.19 & 1.11 & 0.56 & 6.22 & 1.55 \\
\hline 21 & 16.96 & 0.85 & 0.27 & -2.69 & 4.78 & 87 & 14.39 & 0.58 & 0.17 & 6.45 & 1.54 \\
\hline 22 & 16.20 & 0.78 & 0.28 & -0.23 & 4.67 & 88 & 13.48 & 0.18 & -0.24 & 3.36 & 1.46 \\
\hline 23 & 15.46 & 0.77 & 0.21 & -2.21 & 4.49 & 89 & 13.83 & 0.44 & -0.03 & 2.98 & 1.38 \\
\hline 24 & 16.82: & $1.50:$ & $0.85::$ & 2.59 & 4.40 & 91 & 17.23 & 0.81 & $0.04:$ & 5.71 & 1.04 \\
\hline 90 & 19.15 & 1.15 & $0.63::$ & 2.20 & 1.21 & 92 & $(10.42$ & 0.06 & $-0.71)$ & 5.44 & 0.98 \\
\hline 25 & 16.25 & 1.19 & $0.76:$ & 2.93 & 4.38 & 93 & 12.42 & 0.15 & -0.45 & 3.18 & 0.98 \\
\hline 26 & 17.07 & 0.84 & 0.25 & 0.83 & 4.37 & 94 & 15.13 & 0.77 & 0.23 & 3.28 & 0.93 \\
\hline 27 & 16.01 & 1.10 & 0.96 & -0.40 & 4.37 & 95 & 12.61 & 0.20 & -0.22 & 3.72 & 0.90 \\
\hline 28 & 17.13 & 0.84 & 0.36 & -2.28 & 4.29 & 96 & 16.01 & 0.65 & 0.15 & 3.90 & 0.78 \\
\hline 29 & 17.03 & 0.99: & $0.64::$ & -0.08 & 3.93 & 97 & 17.26 & 0.87 & 0.28 & 3.83 & 0.75 \\
\hline 30 & 15.98 & 0.91 & 0.35 & 2.64 & 3.82 & 98 & 14.91 & 1.24 & 0.82 & 4.97 & 0.66 \\
\hline 31 & 15.28 & 1.34 & 1.29 & -2.31 & 3.64 & 99 & 13.58 & 1.42 & $1.52:$ & 2.49 & 0.52 \\
\hline 32 & 14.39 & 0.26 & 0.12 & -2.00 & 3.59 & 100 & 16.16 & 0.93 & 0.44 & 4.65 & 0.51 \\
\hline 33 & 13.72 & 1.45 & 1.79 & 1.33 & 3.48 & 101 & 15.61 & 0.88 & 0.38 & 2.12 & 0.44 \\
\hline 34 & 15.36 & 1.13 & 1.06 & -0.61 & 3.22 & 102 & 16.66 & 0.88 & 0.29 & 2.39 & 0.33 \\
\hline 35 & 15.82 & 1.00 & 0.68 & 2.08 & 3.17 & 103 & 14.96 & 0.34 & 0.18 & 2.26 & 0.25 \\
\hline 36 & 14.72 & 0.80 & 0.35 & 1.31 & 3.11 & 104 & 15.25 & 0.76 & 0.24 & 6.33 & 0.21 \\
\hline 37 & 15.93 & 1.47 & 1.50 & -0.30 & 2.96 & 105 & 15.02 & 0.37 & 0.20 & 1.91 & 0.19 \\
\hline 38 & 15.40 & 0.67 & 0.18 & 0.84 & 2.94 & 106 & 15.67 & 0.77 & 0.23 & 2.17 & 0.04 \\
\hline 39 & 17.21 & 1.13 & 1.05: & 1.62 & 2.88 & 107 & 15.83 & 1.27 & 0.91 & 3.40 & -0.01 \\
\hline 40 & 16.84 & 0.75 & 0.38 & -1.75 & 2.83 & 108 & 16.08 & 0.75 & 0.06 & 6.60 & -0.09 \\
\hline 41 & 15.84 & 0.67 & $0.15:$ & 2.42 & 2.83 & 109 & 16.15 & 0.90 & $0.11:$ & 6.43 & -0.15 \\
\hline 42 & 16.70 & 0.70 & $0.09:$ & 1.51 & 2.82 & 110 & 16.62: & 1.30 & $0.89:$ & 1.16 & -0.24 \\
\hline 43 & 17.10 & 0.85 & 0.38 & -0.67 & 2.81 & 111 & 16.80 & 1.28 & 0.86 & 2.32 & -0.38 \\
\hline 44 & 16.70 & 1.32 & $1.05::$ & 2.02 & 2.63 & 112 & 16.69 & 0.86 & 0.27 & 3.62 & -0.39 \\
\hline 45 & 15.63 & 0.72 & 0.15 & 2.47 & 2.56 & 113 & 15.62 & 1.35 & 0.97 & 6.04 & -0.39 \\
\hline 46 & 14.71 & 0.47 & 0.27 & 1.87 & 2.52 & 114 & 16.64 & 0.90 & 0.34 & 1.12 & -0.47 \\
\hline 47 & 15.46 & 0.59 & 0.34 & -0.46 & 2.49 & 115 & 15.16 & 1.29 & 0.95 & 1.27 & -0.48 \\
\hline 48 & 17.34 & 0.97 & $0.63:$ & -2.33 & 2.49 & 116 & 15.11 & 0.68 & 0.17 & 4.68 & -0.61 \\
\hline 49 & 13.95 & 0.91 & 0.64 & -0.59 & 2.45 & 117 & 15.39 & 0.76 & 0.22 & 5.18 & -0.68 \\
\hline 50 & 16.52 & 1.07 & 0.49 & 2.59 & 2.40 & 118 & 16.75 & 1.03 & 0.49 & 1.49 & -0.70 \\
\hline 51 & 14.89 & 1.13 & 0.79 & 2.56 & 2.35 & 119 & 15.09 & 0.39 & $0.26:$ & 2.41 & -0.87 \\
\hline 52 & 13.02 & 1.17 & 1.01 & 0.24 & 2.33 & 120 & 13.75 & 0.81 & $0.31:$ & 4.33 & -0.90 \\
\hline 53 & 17.09 & 0.76 & 0.15 & -2.38 & 2.33 & 121 & 17.05 & 1.21 & 0.75 & 3.83 & -1.02 \\
\hline 54 & 13.73 & 0.19 & -0.20 & -0.94 & 2.31 & 122 & 16.84 & 0.91 & 0.37 & 2.37 & -1.13 \\
\hline 55 & 14.33 & 0.68 & 0.17 & -1.36 & 2.04 & 123 & 14.72 & 0.79 & 0.24 & 4.09 & -1.15 \\
\hline 56 & 14.39 & 0.24 & -0.09 & -1.91 & 1.91 & 124 & 15.26 & 0.70 & 0.25 & 2.47 & -1.36 \\
\hline 57 & 14.95 & 1.23 & 0.89 & -0.34 & 1.82 & 125 & 16.11 & 1.18 & 1.07 & 2.61 & -1.36 \\
\hline 58 & 16.97 & 0.87 & 0.29 & 1.25 & 1.76 & 126 & 16.06 & 0.73 & $0.06::$ & 6.51 & -1.37 \\
\hline 59 & 15.49 & 0.93 & 0.36 & 2.34 & 1.72 & 127 & 15.32 & 1.06 & 0.80 & 5.95 & -1.37 \\
\hline 60 & 15.17 & 0.70 & 0.41 & 0.98 & 1.72 & 128 & 17.11 & 0.92 & 0.31 & 6.39 & -1.55 \\
\hline 61 & 16.61 & $0.73:$ & 0.12 & 0.53 & 1.68 & 129 & 15.81 & 0.96 & 0.67 & 4.69 & -1.67 \\
\hline 62 & 16.62 & 0.77 & $0.23:$ & 0.64 & 1.66 & 130 & 15.97 & 0.69 & 0.13 & 5.60 & -1.71 \\
\hline 63 & 16.93 & 1.12 & 0.87 & 1.72 & 1.66 & 131 & 16.06 & 0.85 & 0.30 & 4.01 & -2.21 \\
\hline 64 & 16.90 & 0.70 & $0.10:$ & 0.22 & 1.58 & 132 & 13.88 & 0.66 & $0.11:$ & 6.24 & $-2.31(\mathrm{a})$ \\
\hline
\end{tabular}


TABLE 2. (continued)

\begin{tabular}{|c|c|c|c|c|c|c|c|c|c|c|c|}
\hline Star & $V$ & $B-V$ & $U-B$ & $X$ & $Y$ & Star & $V$ & $B-V$ & $U-B$ & $X$ & $Y$ \\
\hline 133 & 16.45 & 0.78 & $0.23:$ & 6.21 & -2.36 & 190 & 16.56 & 1.33 & 0.96 & -6.27 & -1.28 \\
\hline 134 & 15.28 & 0.42 & $0.24:$ & 1.67 & -2.36 & 191 & 13.52 & 0.47 & 0.38 & -2.11 & -1.41 \\
\hline 135 & 13.82 & 0.87 & 0.43 & 4.34 & -2.41 & 192 & 16.86 & 0.95 & 0.56 & -5.02 & -1.53 \\
\hline 136 & 17.22 & 1.21 & 0.90 & 5.39 & -2.54 & 193 & 16.38 & 0.96 & 0.36 & -1.54 & -1.56 \\
\hline 137 & 16.07 & 1.07 & 0.66 & 1.56 & -2.58 & 194 & 11.65 & 0.36 & -0.46 & -6.67 & -1.59 \\
\hline 138 & 16.07 & 1.03 & 0.74 & 3.08 & -2.62 & 195 & 10.89 & 0.18 & -0.56 & -1.74 & -1.66 \\
\hline 139 & 15.94 & 0.69 & 0.16 & 2.63 & -2.68 & 196 & 17.22 & 1.25 & $1.37::$ & -4.08 & -1.76 \\
\hline 140 & 15.15 & 0.74 & 0.18 & 2.37 & -2.77 & 197 & 16.74 & 1.47 & 1.25 & -3.62 & -1.83 \\
\hline 141 & 17.15 & 1.44 & $\ldots$ & 3.95 & $-2.81(\mathrm{a})$ & 198 & 16.15 & 0.87 & 0.30 & -3.90 & -1.84 \\
\hline 142 & 16.55 & 0.77 & 0.06 & 2.77 & -2.82 & 199 & 14.87 & 0.39 & 0.05 & -2.13 & -2.03 \\
\hline 143 & 17.18 & 0.83 & 0.26 & 2.15 & -2.84 & 200 & 17.32 & 1.16 & $0.63::$ & -1.98 & -2.24 \\
\hline 144 & 16.13 & 1.08 & 0.48 & -0.11 & 0.73 & 201 & 16.42 & 0.61 & 0.30 & -1.75 & -2.26 \\
\hline 145 & 16.56 & 0.67 & 0.29 & 0.40 & 0.70 & 202 & 16.79 & 0.92 & 0.39 & -5.88 & -2.34 \\
\hline 146 & 15.27 & 1.45 & $\ldots$ & -0.44 & 0.69 & 203 & 15.48 & 0.51 & 0.36 & -3.47 & -2.38 \\
\hline 147 & 15.37 & 0.44 & 0.29 & -0.02 & 0.57 & 204 & 16.65 & 1.12 & 0.55 & -1.67 & -2.40 \\
\hline 148 & 17.38 & 0.82 & $\ldots$ & -0.59 & 0.56 & 205 & 16.03 & 0.78 & 0.35 & -3.43 & -2.68 \\
\hline 149 & 13.92 & 0.37 & 0.12 & 0.08 & 0.46 & 206 & 15.47 & 0.76 & 0.20 & -1.71 & -2.69 \\
\hline 150 & 13.55 & 0.27 & -0.20 & 0.39 & 0.36 & 207 & 15.49 & 0.57 & 0.26 & -4.93 & -2.91 \\
\hline 151 & 13.95 & 0.30 & -0.05 & -0.51 & 0.24 & 208 & 12.67 & 1.12 & 0.83 & -4.72 & -3.01 \\
\hline 152 & 12.64 & 0.19 & -0.46 & -0.12 & 0.23 & 209 & 16.32 & 0.94 & 0.48 & -2.60 & -3.14 \\
\hline 153 & 16.69 & $1.22::$ & $\ldots$ & -1.18 & 0.21 & 210 & 13.73 & 1.08 & 0.66 & -6.82 & -3.31 \\
\hline 154 & 14.81 & 0.96 & 0.37 & 0.32 & $0.18(b)$ & 211 & 16.52 & 0.88 & 0.30 & -2.57 & -3.41 \\
\hline 155 & 14.83 & 0.99 & $0.71:$ & -0.53 & 0.02 & 212 & 14.73 & 0.90 & 0.32 & -0.49 & -0.41 \\
\hline 156 & 16.36 & 0.91 & $0.47::$ & -0.62 & -0.04 & 213 & 12.12 & 0.17 & -0.43 & -0.80 & -0.47 \\
\hline 157 & 14.90 & 0.38 & -0.01 & 0.33 & $-0.19(\mathrm{c})$ & 214 & 11.23 & 0.15 & -0.59 & -0.66 & -0.59 \\
\hline 158 & 16.57 & 0.80 & 0.24 & -5.06 & 1.89 & 215 & 13.43 & 0.39 & -0.20 & 0.26 & -0.72 \\
\hline 159 & 13.73 & 0.29 & -0.12 & -3.35 & 1.69 & 216 & 12.45 & 0.40 & 0.29 & 0.70 & -1.03 \\
\hline 160 & 15.30 & 2.13 & $\ldots$ & -4.23 & 1.50 & 217 & 15.71 & 0.96 & 0.64 & 0.40 & -1.09 \\
\hline 161 & (11.35 & 0.16 & $-0.58)$ & -4.64 & 1.44 & 218 & 13.88 & 0.30 & 0.04 & -0.64 & -1.20 \\
\hline 162 & 17.10 & 1.12 & $0.98:$ & -3.97 & 1.28 & 219 & 17.06 & 1.02 & $0.60:$ & -1.09 & -1.54 \\
\hline 163 & 16.46 & 0.74 & 0.33 & -4.56 & 0.90 & 220 & 13.51 & 0.67 & 0.48 & -1.18 & -1.82 \\
\hline 164 & 16.56 & 0.75 & 0.21 & -4.94 & 0.84 & 221 & 11.89 & 0.45 & 0.25 & -0.87 & -2.38 \\
\hline 165 & 14.64 & 0.28 & 0.16 & -3.20 & 0.82 & 222 & 16.14 & $2.49::$ & $\ldots$ & 0.52 & -2.81 \\
\hline 166 & 15.61 & 0.55 & 0.33 & -2.94 & 0.70 & 223 & 15.41 & 1.01 & 0.43 & 2.67 & -3.33 \\
\hline 167 & 14.87 & 0.32 & 0.21 & -2.04 & 0.68 & 224 & 14.33 & 0.74 & 0.20 & -0.57 & -3.42 \\
\hline 168 & 16.29 & 0.71 & 0.29 & -1.90 & 0.46 & 225 & 14.48 & 0.69 & 0.19 & -1.21 & -3.51 \\
\hline 169 & 15.60 & 0.67 & 0.16 & -2.61 & 0.46 & 226 & 16.89 & $0.97:$ & 0.60 : & -0.03 & -3.51 \\
\hline 170 & 17.18 & 0.70 & 0.28 & -3.03 & 0.42 & 227 & 15.90 & 0.84 & 0.33 & -1.42 & -3.52 \\
\hline 171 & 15.65 & 0.60 & 0.07 & -3.97 & 0.27 & 228 & 16.63 & 1.18 & $0.96:$ & 1.69 & -3.68 \\
\hline 172 & 14.83 & 0.76 & 0.22 & -1.62 & 0.24 & 229 & 17.24 & 0.98 & $0.36::$ & 2.57 & -3.72 \\
\hline 173 & 16.53 & 1.27 & $0.80:$ & -5.82 & 0.18 & 230 & 16.94 & 0.91 & 0.36 & -0.32 & -3.80 \\
\hline 174 & 16.15 & 1.15 & 0.68 & -1.38 & 0.15 & 231 & 16.02 & 0.86 & 0.27 & -0.08 & -3.81 \\
\hline 175 & 14.35 & 0.87 & 0.40 & -1.43 & -0.05 & 232 & 13.68 & 0.74 & 0.18 & 2.49 & -3.98 \\
\hline 176 & 16.02 & 1.55 & $1.32::$ & -6.27 & -0.11 & 233 & 13.47 & 0.72 & 0.15 & 1.64 & -4.01 \\
\hline 177 & 17.05 & 0.86 & $0.29:$ & -5.92 & -0.31 & 234 & 13.37 & 0.49 & 0.34 & 1.06 & -4.08 \\
\hline 178 & 17.21 & 1.08 & 0.75 & -5.28 & -0.34 & 235 & 14.67 & 0.84 & 0.35 & 0.26 & -4.21 \\
\hline 179 & 13.53 & 0.50 & 0.34 & -4.86 & -0.36 & 236 & 13.91 & 0.37 & 0.27 & 2.20 & -4.27 \\
\hline 180 & 14.24 & 0.35 & 0.25 & -3.02 & -0.37 & 237 & 11.44 & 0.29 & 0.22 & 0.46 & -4.56 \\
\hline 181 & 16.11 & 0.76 & 0.20 & -2.61 & -0.43 & 238 & 15.28 & 0.99 & 0.67 & -1.02 & -4.61 \\
\hline 182 & 15.85 & 0.77 & 0.27 & -3.18 & -0.50 & 239 & 16.30 & 0.90 & 0.44 & -0.24 & -4.64 \\
\hline 183 & 16.31 & 1.44 & 1.14 & -2.07 & -0.60 & 240 & 14.87 & 0.69 & 0.14 & 1.96 & -4.79 \\
\hline 184 & 16.85 & 0.75 & 0.20 & -3.72 & -0.81 & 241 & 16.03: & $0.82::$ & $0.36::$ & 1.06 & -5.09 \\
\hline 185 & 15.63 & 0.72 & 0.18 & -5.10 & -0.91 & 242 & 16.02 & 1.05 & 0.69 & -2.60 & -5.15 \\
\hline 186 & 13.85 & 0.23 & -0.18 & -5.29 & -1.08 & 243 & 15.36 & 0.74 & 0.22 & -0.77 & -5.24 \\
\hline 187 & 15.22 & 0.70 & 0.40 & -1.75 & -1.10 & 244 & 13.55 & 1.37 & 1.39 & -2.04 & -5.29 \\
\hline 188 & 15.19 & 0.57 & 0.30 & -2.29 & -1.11 & 245 & 13.57: & $0.61:$ & $0.20:$ & 2.81 & -5.55 \\
\hline 189 & 17.24 & 0.98 & 0.48 & -3.11 & -1.25 & 246 & 13.62 & 1.27 & $1.07::$ & 2.44 & -5.88 \\
\hline
\end{tabular}

Notes: (a) double.
(b) HD 5005f
(c) HD 5005g

In general there is excellent agreement of the color excess ratios $\mathrm{E}_{U-B} / \mathrm{E}_{B-V}$ for cluster stars with those derived for stars in the surrounding field, as well as with the trend established for stars in other fields near the same location in the Galaxy (Turner 1976b; Turner et al. 1992). There are four anomalous objects in the cluster sample: stars $150,151,214$, and 215, all of which are recognized Be stars. Star 214 may have $\mathrm{H}^{-}$free-bound continuum radiation superimposed upon its stellar continuum; it appears to have an ultraviolet excess of $\delta(U-B)=0.06$. Stars 150,151 , and 215 are rapidly- 
TABLE 3. Additional field stars lying within 0.5 of HD 5005.

\begin{tabular}{llllllllll}
\hline \hline $\mathrm{HD}(\mathrm{E})$ & $\mathrm{BD}$ & Sp. Type & Ref. & $V$ & $B-V$ & $U-B$ & $n$ \\
\hline 4742 & $+55^{\circ}$ & 171 & G5 & $\mathrm{a}$ & 8.42 & 0.61 & +0.13 & 3 \\
4787 & $+55^{\circ}$ & 173 & B7V & $\mathrm{b}$ & 8.73 & 0.04 & -0.29 & 3 \\
236552 & $+55^{\circ}$ & 175 & KI III & $\mathrm{d}$ & 8.25 & 1.20 & +1.17 & 3 \\
4960 & $+55^{\circ}$ & 188 & K5 III & $\mathrm{e}$ & 7.85 & 1.52 & +1.72 & 3 \\
236564 & $+55^{\circ}$ & 189 & K2 III & $\mathrm{e}$ & 9.47 & 1.33 & +1.28 & 3 \\
$\ldots$ & $+55^{\circ}$ & 194 & F1 & $\mathrm{c}$ & 10.43 & 0.41 & +0.08 & 3 \\
5177 & $+55^{\circ}$ & 195 & A9V & $\mathrm{b}$ & 7.14 & 0.28 & +0.12 & 3 \\
236572 & $+55^{\circ}$ & 196 & F5 & $\mathrm{a}$ & 9.80 & 0.57 & +0.10 & 3 \\
236573 & $+55^{\circ}$ & 198 & G0 & $\mathrm{a}$ & 8.65 & 0.60 & +0.08 & 3 \\
$\ldots$ & $+55^{\circ}$ & 199 & gK3 & $\mathrm{c}$ & 10.20 & 1.38 & +1.46 & 4 \\
236575 & $+55^{\circ}$ & 200 & G8 III-IV & $\mathrm{d}$ & 8.08 & 1.03 & +0.75 & 3 \\
$\ldots$ & $+55^{\circ}$ & 203 & G9 & $\mathrm{c}$ & 10.31 & 1.10 & +0.87 & 3 \\
$\ldots$ & $+55^{\circ}$ & 204 & F1 & $\mathrm{c}$ & 10.36 & 0.38 & +0.04 & 3 \\
$(\mathrm{ADS} 694 \mathrm{~A})$ & $+56^{\circ} 139 \mathrm{~A}$ & gK0 & $\mathrm{c}$ & 9.76 & 1.17 & +1.02 & 4 \\
236559 & $+56^{\circ}$ & 140 & K5 III & $\mathrm{e}$ & 8.93 & 1.22 & +1.21 & 3 \\
5136 & $+56^{\circ}$ & 144 & F1 V & $\mathrm{b}$ & 7.41 & 0.35 & +0.03 & 3 \\
$\ldots$ & $+56^{\circ}$ & 146 & G2 & $\mathrm{c}$ & 9.92 & 0.64 & +0.11 & 3 \\
236574 & $+56^{\circ}$ & 147 & A0 & $\mathrm{a}$ & 9.53 & 0.21 & +0.20 & 3 \\
\hline
\end{tabular}

References: (a) Spectral type from $\mathrm{HD}(\mathrm{E})$.

(b) Spectral type from this study.

(c) Spectral type estimated from dereddened colors.

(d) Spectral type from Yoss (1961).

(e) Spectral type from $\mathrm{HD}(\mathrm{E})$ and luminosity class inferred from dereddened colors.

rotating, late B-type stars recently arrived on the main sequence. All three exhibit characteristics that may be associated with equatorial emission rings; they appear to have rather extreme ultraviolet excesses of $\delta(U-B) \approx 0.13-0.25$.

There are 12 cluster $\mathrm{OB}$ stars that do not exhibit anomalous $U-B$ colors. A regression analysis was used to fit a straight line passing through the origin to the data (see Turner 1989), and yielded a reddening slope of $\mathrm{E}_{U-B} / \mathrm{E}_{B-V}=0.735 \pm 0.034$ s.e. A similar analysis of the data for stars in the surrounding field gave $\mathrm{E}_{U-B} / \mathrm{E}_{B-V}=0.736 \pm 0.031$ s.e., while an analysis of the combined data produced a slope of $\mathrm{E}_{U-B} / \mathrm{E}_{B-V}=0.735$ \pm 0.032 s.e. The adopted interstellar reddening relation of $\mathrm{E}_{U-B} / \mathrm{E}_{B-V}=0.735$ for OB stars in the IC 1590 field is in good agreement with values of $0.74-0.76$ that would be predicted from the extinction properties of dust in nearby galactic fields. The extinction towards IC 1590 is therefore characterized by fairly normal dust properties in the visual spectral region.

In 1984 September, near-infrared observations were obtained for the five brightest early-type cluster members using the InSb Autophote System with standard $K$ filter attached to the $1.3 \mathrm{~m}$ reflector at KPNO. NOAO in-house standards, which are very close o the original Johnson $(1965,1966)$ system, were used for calibration. Extinction in the $V-K$ and $B-V$ bandpasses was calculated by comparing the observed colors with the intrinsic values corresponding to the derived spectral types. The ratios of total to selective absorption for the five stars were then calculated by scaling the parameters for van de Hulst's curve no. 15 (see Johnson 1968), namely, using

$$
R_{V}(K)=1.10\left[\mathrm{E}_{V-K} / \mathrm{E}_{B-V}\right] .
$$

The results, summarized in Table 4 , imply an anomalous value of $R_{V}(K)=3.67 \pm 0.22$ s.d. for the five observed stars.

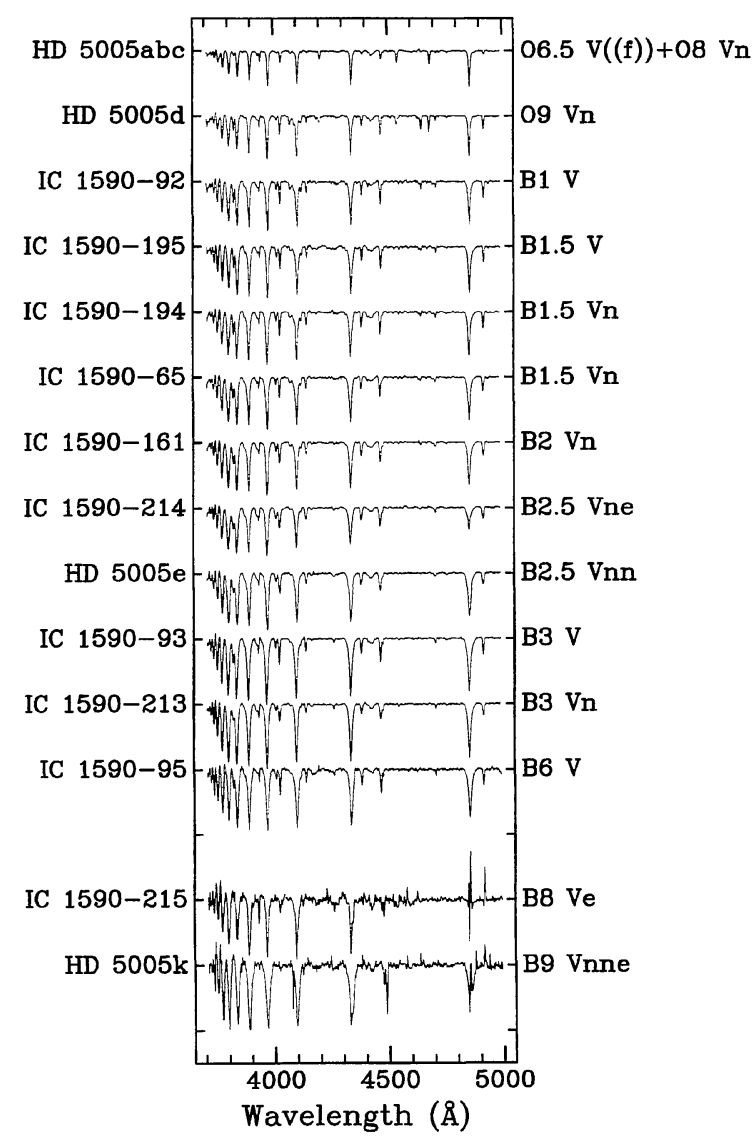

FIG. 3. A mosaic of continuum-normalized CCD spectra for 14 of the bright stars in IC 1590. The stars are identified at left, while their spectral types are given at right. The spectra are offset from one another by 0.5 in continuum intensity, except between the spectra for stars 95 and 215 , where an offset of 1.0 has been used because of the strength of the emission in the latter.

If the results for stars HD 5005abc, 65 and 214 are discounted owing to the possibility that intrinsic infrared emission is associated with the stars (all of which are rapid rotators and one a known emission-line object), the average for the remaining two cluster stars, which are otherwise normal dwarfs, is $R_{V}(K)=3.45 \pm 0.11$ s.d.

It was considered advantageous to verify the $K$-band results with measures in another red bandpass. Thus, in 1985 September the data were augmented by $V-I$ observations on the Kron-Cousins system (Bessel 1979) for 10 cluster stars. They were obtained with the Ritchey-Chretien reflector at the U. S. Naval Observatory, the same cooled EMI 9658R photomultiplier as in Section 2, and appropriate filters. The observational results are presented in Table 5, where the intrinsic $V-I$ colors used for inferring color excesses are those of Johnson (1968) transformed to the Kron-Cousins system by means of the empirical relationships of Fernie (1983). They agree closely with the intrinsic $(V-I)_{\mathrm{KC}}$ colors for early-type stars tabulated by Guetter \& Vrba (1989). The last column of the table lists the values of $R_{V}(I)$ calculated from the relation

$$
R_{V}(I)=2.40\left[\mathrm{E}_{V-I} / \mathrm{E}_{B-V}\right],
$$




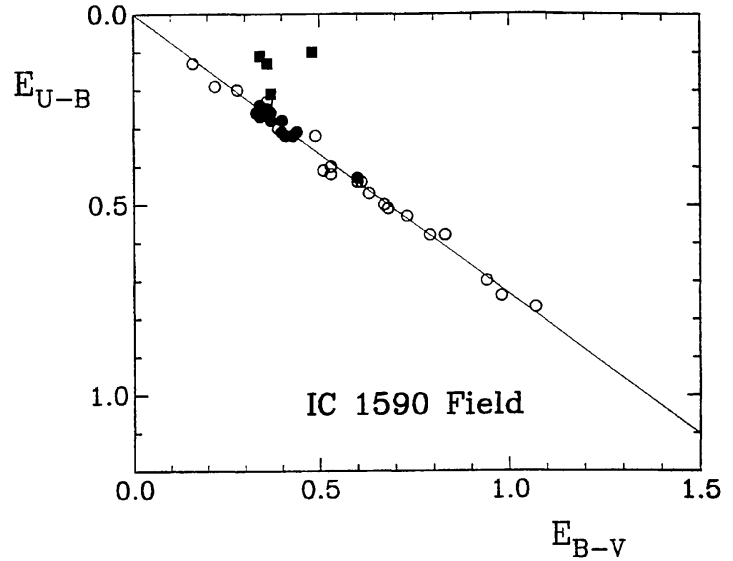

FIG. 4. A plot of color excesses $\mathrm{E}_{U-B}$ and $\mathrm{E}_{B-V}$ for stars in IC 1590 (filled symbols) and the surrounding field (open symbols). Filled squares designate the four cluster Be stars which exhibit ultraviolet excesses. The plotted line has a slope $\mathrm{E}_{U-B} / \mathrm{E}_{B-V}=0.735$.

which applies to van de Hulst's curve no. 15 (Johnson 1968) when transformed to the Kron-Cousins system using Fernie's relations. If star 214 is rejected on the basis of its emissionline characteristics, the mean value for the remaining 9 stars is $R_{V}(I)=3.44 \pm 0.13$ s.d. Guetter \& Vrba (1989) find that the constant in the above relationship should be 2.45 rather than 2.40. However, the resulting value of $R_{V}(I)$ changes very little - to $R_{V}(I)=3.45 \pm 0.14$ s.d.-when their precepts are adopted.

Both the $K$-band data and $I$-band data imply that anomalous extinction exists in the region of IC 1590, and one might be tempted to suggest that the anomaly is associated with the dust in the cluster itself. However, a curious property of the $R_{V}(I)$ values is that they do not exhibit a dependence on reddening, as is also evident from the plot of color excesses $\mathrm{E}_{V-I}$ versus $\mathrm{E}_{B-V}$ shown in Fig. 5. The trend of color excesses depicted here is linear and passes through the origin, features which are more characteristic of a common reddening law over all color excesses than of a different relation applicable to the foreground reddening that is contaminated by an anomalous component valid for the cluster dust itself. Moreover, the reddening line slope of $\mathrm{E}_{U-B} / \mathrm{E}_{B-V}=0.735$ that applies to the extinction of stars in the cluster and surrounding field would be characterized by a value of $R_{V}=3.0$ if associated with nearby dust clouds (Turner 1994, 1996a, 1996b), whereas anomalous extinction with $R_{V}=3.44-3.67$ would be associated with very small extinction ratios of $\mathrm{E}_{U-B} / \mathrm{E}_{B-V}=0.52-0.60$. There is no evidence in the reddening data for spectroscopically-observed cluster stars (Fig. 4) for an abrupt reduction in the reddening slope

TABLE 4. $R_{V}(K)$ determination for five members of IC 1590 .

\begin{tabular}{llccccccc}
\hline \hline \multicolumn{1}{c}{ Star } & Sp. Type & $V$ & $B-V$ & $V-K$ & $n(K)$ & $E_{B-V}$ & $E_{V-K}$ & $R_{V}(K)$ \\
\hline $5005 \mathrm{abc}$ & O6.5 V((f)) & 7.93 & 0.10 & 0.56 & 1 & 0.42 & 1.50 & 3.92 \\
65 & B1.5 Vn & 10.78 & 0.09 & 0.38 & 2 & 0.34 & 1.15 & 3.71 \\
92 & $2 \mathrm{~B} 1 \mathrm{~V}$ & 10.42 & 0.06 & 0.22 & 2 & 0.32 & 1.03 & 3.53 \\
195 & $\mathrm{~B} 1.5 \mathrm{~V}$ & 10.87 & 0.18 & 0.55 & 2 & 0.43 & 1.32 & 3.37 \\
214 & B2.5 Vne & $11.26:$ & 0.15 & 0.61 & 1 & 0.37 & 1.28 & 3.80 \\
\hline \hline
\end{tabular}

TABLE 5. $R_{V}(I)$ determination for ten members of IC 1590 .

\begin{tabular}{llccccccc}
\hline \hline Star & Sp. Type & $V$ & $B-V$ & $V-I M$ & $n(I)$ & $E_{B-V}$ & $E_{V-I}$ & $R_{V}(I)$ \\
\hline $5005 \mathrm{abc}$ & O6.5 V((f)) & 7.93 & 0.10 & 0.22 & 1 & 0.42 & 0.57 & 3.26 \\
$5005 \mathrm{~d}$ & O9 Vn & 9.78 & 0.06 & 0.21 & 3 & 0.37 & 0.55 & 3.57 \\
65 & Bl.5 Vn & 10.78 & 0.09 & 0.22 & 3 & 0.34 & 0.49 & 3.47 \\
92 & B1 V & 10.42 & 0.06 & 0.18 & 3 & 0.32 & 0.46 & 3.46 \\
93 & B3 V & 12.40 & 0.16 & 0.34 & 3 & 0.36 & 0.55 & 3.67 \\
161 & B2 Vn & 11.35 & 0.16 & 0.30 & 3 & 0.40 & 0.56 & 3.37 \\
194 & B1.5 Vn & 11.65 & 0.35 & 0.59 & 3 & 0.60 & 0.86 & 3.45 \\
195 & B1.5 V & 10.87 & 0.18 & 0.33 & 3 & 0.43 & 0.60 & 3.36 \\
213 & B3 Vn & 12.12 & 0.17 & 0.30 & 3 & 0.37 & 0.51 & 3.31 \\
214 & B2.5 Vne & $11.26:$ & 0.15 & 0.40 & 3 & 0.37 & 0.63 & 4.09 \\
\hline \hline
\end{tabular}

within the cluster proper, which implies that the extinction law within IC 1590 must also apply to the foreground reddening as well.

Infrared excesses for stars can be attributed to several factors (see Mendoza 1968; Turner 1994), including an anomalous extinction law characterized by $R_{\mathrm{V}}>3$, intrinsic infrared emission, or, for the near-infrared spectral region, normal variations in the standard extinction law around the Galaxy. The value of $\mathrm{E}_{V-I} / \mathrm{E}_{B-V}=1.425 \pm 0.047$ s.e. for stars in IC 1590 is larger than typical values (on the Kron-Cousins system) which range from 1.2 to 1.4 , as transformed from Johnson's (1968) results for galactic fields that exhibit relatively normal extinction laws. Such a steep reddening slope in the near infrared should correspond to a somewhat largerthan-normal $R$ value, as also implied by the $R_{V}(K)$ data. The mean value of $R_{V}(K)=3.45$ for the two objects for which intrinsic infrared emission seems unlikely is identical to the calculated mean value for $R_{V}(I)$. The result is also supported by a variable-extinction analysis of cluster stars.

Figure 6 is a $U B V$ color-color diagram for all stars in IC 1590 and its environs for which we tabulate photometry. The intrinsic relation depicted is the locus of intrinsic colors for

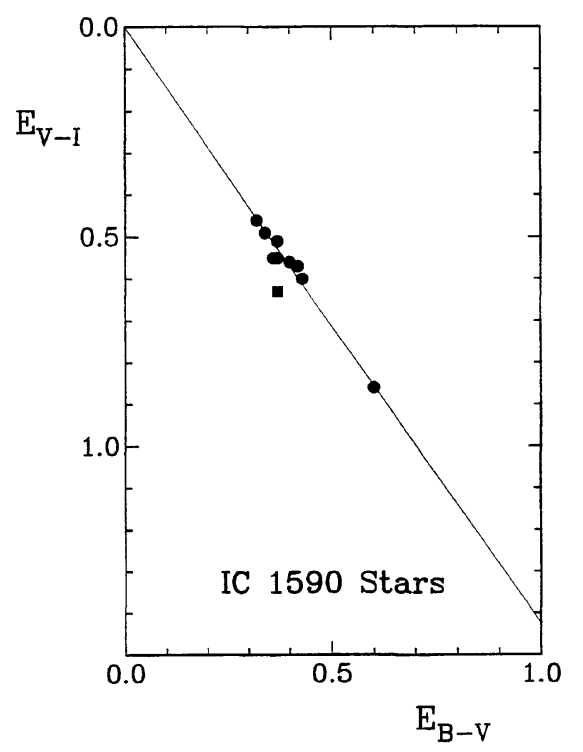

FIG. 5. A plot of color excesses $\mathrm{E}_{V-I}$ and $\mathrm{E}_{B-V}$ for stars in IC 1590. The filled square designates star 214 , a rapidly-rotating Be star. The plotted line has a slope $\mathrm{E}_{V-I} / \mathrm{E}_{B-V}=1.425$. 


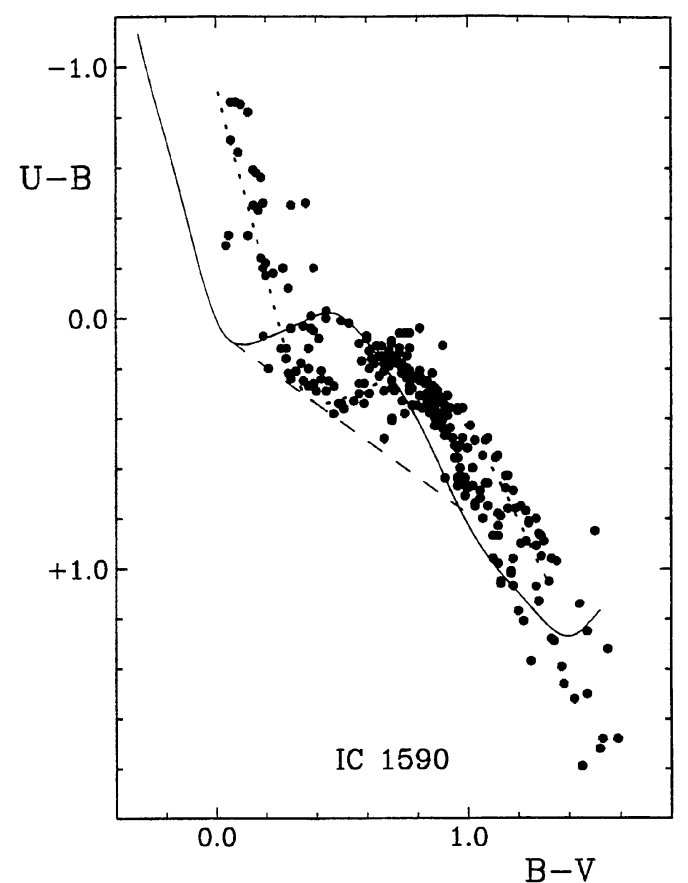

FIG. 6. Color-color diagram for stars in IC 1590. The intrinsic relation for main-sequence stars (solid curve) is also depicted for a reddening of $\mathrm{E}_{B-V}=0.32$ (dotted curve) using a reddening relation of slope $\mathrm{E}_{U-B} / \mathrm{E}_{B-V}=0.735$ (depicted by a dashed line originating with the intrinsic colors for A2 stars).

O and B stars (Johnson 1966; FitzGerald 1970), the zero-age zero-rotation main sequence locus for $\mathrm{A}$ and $\mathrm{F}$ stars (Turner 1996b), and the intrinsic color relation for Pleiades G and K dwarfs (Turner 1979). Two features of the data stand out, namely interstellar reddening of cluster stars is generally small and a small amount of differential reddening is present in the field. The same conclusions can be made from the data of Figs. 4 and 5. The reddening of cluster $\mathrm{O}$ and B-type stars is simple and easy to establish from their $U B V$ colors, particularly when they are used in conjunction with an optical image of the field and a plot of the space reddening across it. The Be stars are exceptions, but they can be dereddened from their spectral types. Also, cluster mainsequence members of spectral type $\mathrm{A} 0$ or later seem to be absent, so incorrect assignments of interstellar reddening, while unlikely, are unimportant for a variable-extinction analysis.

Figure 7 is a plot of apparent distance modulus as a function of color excess-a variable-extinction diagram-for all stars in the field of IC 1590 that could be dereddened from the available $U B V$ data, either to the nearest portion of the intrinsic color relation or in conjunction with available spectroscopic and other reddening information. Luminosities for stars with spectral types were established from the calibrations of Keenan (1963) for late-type stars and Turner (1980) for early-type stars, and the zero-age main sequence (ZAMS) of Turner (1976a, 1979) was used to assign absolute magnitudes to all other (presumed main-sequence) stars on the basis of their derived intrinsic colors. The situation for $\mathrm{G}$ and $\mathrm{K}$ dwarfs is complicated by the fact that their $U B V$ colors can

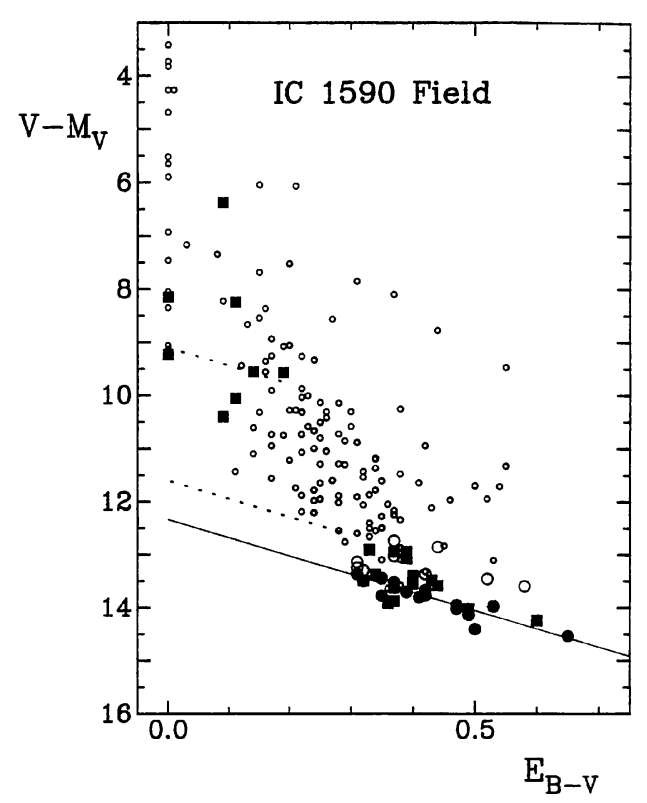

FIG. 7. Variable-extinction diagram for IC 1590 stars. Filled and open large circles denote likely cluster members (filled symbols represent ZAMS objects) and small open circles denote probable field stars, as identified from ZAMS-fitting, while filled squares represent spectroscopically-observed stars ( $\mathrm{K}$ giants and cluster $\mathrm{OB}$ stars). The best-fitting line of slope $R=3.44$ is a lower envelope fit for true ZAMS members of the cluster. Dotted lines of identical slope are used to identify the distance moduli corresponding to sudden increases in field reddening.

be affected by both reddening and metallicity variations. A few late-type stars lying close to the intrinsic relation were therefore judged to be unreddened, and their luminosities were adjusted for possible metallicity effects using their inferred ultraviolet excesses (see Turner et al. 1994). The situation for late-type stars is further complicated by the fact that, for a magnitude-limited sample, many of the objects are field G and K giants (see McCuskey 1965) that have erroneously been assigned main-sequence luminosities for lack of spectroscopic information on their true luminosities. Such stars produce much of the scatter in the upper portion of Fig. 7, and, unless taken into account, may lead to erroneous interpretations about the run of reddening with distance for the cluster field.

A detailed variable-extinction analysis is not simply a matter of calculating reddenings and apparent distance moduli for an entire stellar sample by means of guesswork, but is an interactive procedure that begins with the data for stars with the most well-established reddenings and luminosities and continues by adding other stars while testing their derived parameters against the results for the initial sample. The methods employed are otherwise those described in Turner et al. (1992) and Turner (1992), namely, the use of spectral type-dependent reddening slopes standardized to the results for early-type stars (Fig. 4) and color excesses $\mathrm{E}_{B-V}$ normalized to those for B0 stars seen through the same amount of extinction. The distance moduli for stars without spectral types are only minimum values, since binarity and evolution away from the ZAMS make the true ab- 


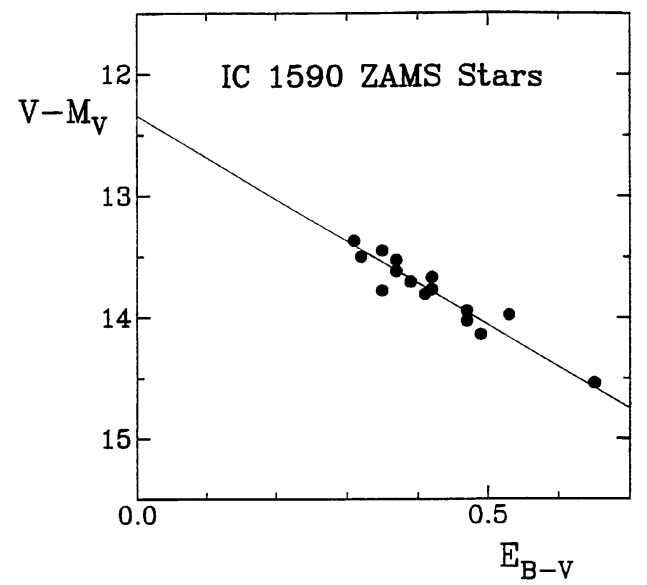

FIG. 8. An enlargement of Fig. 7 for the 15 ZAMS stars used to establish $R_{V}=A_{V} / E_{B-V}$. The line has slope $R=3.44$

solute magnitudes for such stars more negative than their ZAMS values.

The trends in the data points of Figs. 6 and 7 suggest a number of important conclusions about the extinction towards IC 1590 . First, there appears to be little or no reddening out to a distance of about $660 \mathrm{pc}\left(V_{0}-M_{V}=9.1\right)$, beyond which most stars are reddened by $\mathrm{E}_{B-V} \approx 0.15$ or more. A second increase in reddening occurs at a distance of around $2.0-2.2 \mathrm{kpc}\left(V_{0}-M_{V}=11.5-11.7\right)$, and, according to the spatial location of affected stars, may be associated with the dust complex containing the molecular cloud to the southwest of the cluster. Members of IC 1590 itself, including likely pre-main-sequence stars, display a minimum reddening of $\mathrm{E}_{B-V} \approx 0.32$, and are readily distinguished from field stars by that criterion.

The distribution of color excesses for cluster stars is somewhat small for a reliable determination of $R_{V}=A_{V} / E_{B-V}$, but the excellent quality of the photometric data keeps the uncertainties in $V-M_{V}$ established from ZAMS values small, of order $\pm 0^{\mathrm{m}} 1$ (much smaller than the values of \pm 0 m 5 typical of spectroscopic distance moduli). The data for the 15 stars that best define the cluster ZAMS are shown separately in Fig. 8. Least-squares and nonparametric fits to the data yield a best value of $R_{V}=3.44 \pm 0.16$ for the cluster, in quite good agreement with the $R_{V}(K)$ and $R_{V}(I)$ results.

The formal mean of the three estimates for $R_{V}$ by $K$-band photometry, $I$-band photometry $[$ for which $R_{V}(I)=3.44$ was adopted], and a variable-extinction analysis of the $U B V$ data is $\left\langle R_{V}\right\rangle=3.44 \pm 0.07$, which we adopt for the dust extinction towards IC 1590. It is not clear if any of the extinction arises within the cluster and its $\mathrm{H}$ II region. Most of the variations in space reddening across the field of IC 1590 are fairly smooth and can be accounted for by variations in the density of foreground dust, much of which must belong to the dust complex roughly $2.0-2.2 \mathrm{kpc}$ distant. Nor is IC 1590 an isolated case of abnormal extinction with $R_{V}>3.0$ for dust lying in the region of the Perseus spiral arm. Similar anomalies are presented by Turner (1976b) and Turner et al. (1992), namely, NGC $129\left(R_{V}=3.20 \pm 0.30\right)$,
NGC $1039\left(R_{V}=3.43 \pm 0.24\right)$, and NGC $1893\left(R_{V}=3.30\right.$ $\pm 0.10)$. Since the visual extinction laws for those regions and IC 1590 are otherwise normal $\left(\mathrm{E}_{U-B} / \mathrm{E}_{B-V}=0.76,0.75\right.$, 0.77 , and 0.735 , respectively), the explanation may lie in the characteristics of the dust towards the Perseus arm, which clearly differs in its infrared extinction properties from that exhibited by nearby dust (Turner 1994, 1996a, 1996b).

\section{STAR COUNTS AND CLUSTER MEMBERSHIP}

Four partially overlapping CCD frames (each containing HD 5005) were obtained during January and August 1993 in the $V$ bandpass with the $1 \mathrm{~m}$ telescope at the U.S. Naval Observatory using TEK $2048 \times 2048$ and TEK $1024 \times 1024$ CCD detectors, respectively. The pixel size for both devices is 0.68 at the image plane, resulting in frame areas of almost $540 \operatorname{arcmin}^{2}$ and $135 \operatorname{arcmin}^{2}$, respectively. When the four frames were aligned in a mosaic, the resulting fields covered $1 / 2$ degrees $^{2}$ and 1/8 degrees ${ }^{2}$ of the cluster, respectively, centered on the trapezium system HD 5005. The observations were again reduced using the DAOPHOT profile-fitting routine NSTAR (Stetson 1987), and the frame co-ordinates were transformed to radial distance from HD 5005. Since the photometry obtained with the TI 800 chip is complete for stars to a visual magnitude of 17.0 , all objects with $V$ $\geqslant 17.0$ were rejected in the TEK frames.

Star counts were made in concentric 1-arcmin-wide quarter-ring segments (centered on HD 5005) on each frame, then averaged and normalized. Since the TEK 1024 detector is smaller, its curvature in the focal plane (and thus the variation in the point spread function across the frame) is less pronounced than that for the TEK 2048 CCD. Therefore, the results obtained with the smaller CCD were used for regions with radial distances from HD 5005 of less than 9 arcmin, and were supplemented by the TEK 2048 observations for larger distances. The combined results are shown in Fig. 9, where the error bars denote the uncertainties in the star counts computed using Poisson statistics.

The analysis of the star count data included the effects of field star contamination as described by van den Bergh \& Sher (1960) and Leonard (1988). A peak in the distribution of Fig. 9 is apparent within 4 arcmin of HD 5005, and, according to the results of Kholopov (1969), we expect a low density corona to extend the true cluster radius to at least 7 arcmin. Potential cluster members were detected that far from HD 5005. The proper background density is difficult to establish because of an increase in the dust obscuration near HD 5005 itself. The background counts therefore appear to increase slightly near the edge of the field. We therefore selected the annulus running between 7 and 15 arcmin from HD 5005 as most representative of the background; the field star density in that region is $1.41 \pm 0.05$ stars $\operatorname{arcmin}^{-2}$. The corresponding number of expected cluster members lying within 7 arcmin of HD 5005 is $58 \pm 17$, and the numbers expected to fall within each of the 1 -arcmin-wide annuli about HD 5005 are $16 \pm 2,15 \pm 4,18 \pm 5,8 \pm 6,0 \pm 6,0 \pm 7$, and $3 \pm 8$, respectively. By way of comparison, our analysis of the photometric data indicates that there are $16,12,14,11$, 4,4 , and 2 likely members, respectively, that fall within 


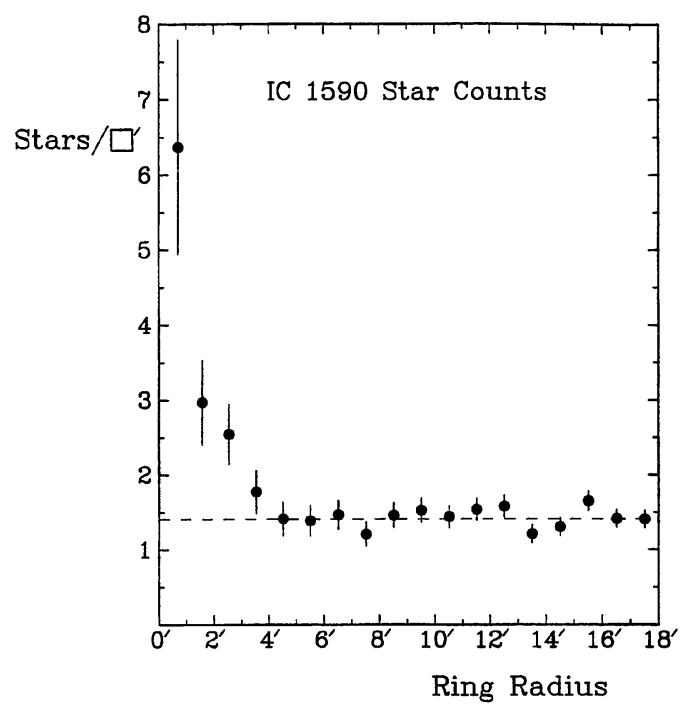

FIG. 9. Star counts for the field of IC 1590 in rings centered on HD 5005 Poisson error bars are indicated, and the dashed line represents the derived field star density of 1.41 stars $\operatorname{arcmin}^{-2}$.

those regions, and 63 likely cluster members in total. The photometrically-selected sample of likely members falls within the limits of the star count predictions.

The selection of likely cluster members was guided by several different criteria, namely the star count predictions, a comparison of the inferred reddenings for stars with the local space reddening exhibited by other likely members, and the location of stars in the variable-extinction diagram (Fig. 7). Faint stars in IC 1590 also appear to concentrate slightly north and west of HD 5005, which may indicate that the geometric center of the cluster is displaced from HD 5005 in that direction. Candidate cluster members were therefore ex- amined with regard to their spatial location relative to HD 5005 , and the best candidates were indeed found to lie preferentially to the northwest of HD 5005. The reduced data for the 62 resolved stars selected as likely cluster members (HD $5005 \mathrm{~b}$ cannot be separated from HD 5005a) are summarized in Table 6 and plotted in Fig. 10. There are 41 likely members of spectral type earlier than A0, and 22 likely members of later spectral type, all of which are pre-main-sequence stars.

The distance to IC 1590 based upon the 15 ZAMS members identified in Fig. 8 is $2.94 \pm 0.15 \mathrm{kpc}$, as inferred from the derived distance modulus of $V_{0}-M_{V}=12.34 \pm 0.11$ s.d. ( \pm 0.03 s.e.) for the same stars, where the calculated uncertainty includes the uncertainty in $R_{V}$. The cluster is clearly quite young. The presence in the trapezium system HD 5005 of $06.5,08$, and 09 stars on the main sequence implies an age of no more than about $3.5 \times 10^{6}$ years, and the 22 premain-sequence stars further restrict that estimate to exactly $3.5( \pm 0.2) \times 10^{6}$ years. Palla \& Stahler $(1993)$ have provided theoretical gravitational isochrones for pre-main-sequence stars of intermediate mass, and the restricted location of likely pre-main-sequence members of IC 1590 in the colormagnitude diagram of Fig. 10 places rather stringent restrictions on the age of those stars. If we attribute the small amount of scatter to age spread, then the cluster contains stars which are $10^{6}$ to $3.5 \times 10^{6}$ years old. However, that presents some difficulties in accounting for the population of the main sequence to spectral type B9.5 $\left(2.2 \mathscr{l}_{\odot}\right)$, since the Palla \& Stahler (1993) evolutionary tracks indicate that only stars of masses greater than about $2.6 \mathscr{O}_{\odot}$ should have reached the ZAMS after $3.5 \times 10^{6}$ years. If we attribute the scatter for pre-main-sequence stars to either binarity or light contamination from circumstellar disks and the presence of B9.5 stars near the ZAMS to rapidly-rotating stars of slightly larger mass viewed nearly pole-on (cf. Maeder \& Peytrem-

TABLE 6. Reduced data for likely members of IC 1590.

\begin{tabular}{lccccccccccc}
\hline \hline Star & $(B-V)_{0}$ & $E_{B-V}{ }^{\mathrm{a}}$ & $V_{0}$ & $\mathrm{Star}$ & $(B-V)_{0}$ & $E_{B-V}{ }^{\mathrm{a}}$ & $V_{0}$ & Star & $(B-V)_{0}$ & $E_{B-V^{\mathrm{a}}}$ & $V_{0}$ \\
\hline $5005 \mathrm{ab}$ & -0.32 & 0.40 & 6.96 & 93 & -0.20 & 0.36 & 11.18 & 165 & -0.03 & 0.32 & 13.54 \\
$5005 \mathrm{c}$ & -0.31 & 0.44 & $7.68:$ & 94 & +0.46 & 0.33 & 13.99 & 167 & -0.02 & 0.35 & 13.67 \\
$5005 \mathrm{~d}$ & -0.31 & 0.37 & 8.51 & 95 & -0.13 & 0.33 & 11.47 & 172 & +0.46 & 0.32 & 13.73 \\
32 & -0.04 & 0.31 & 13.32 & 101 & +0.39 & 0.52 & 13.82 & 173 & +0.76 & 0.55 & 14.64 \\
36 & +0.36 & 0.46 & 13.14 & 103 & 0.04 & 0.39 & 13.62 & 174 & +0.83 & 0.35 & 14.95 \\
44 & +0.95 & 0.41 & 15.29 & 105 & -0.04 & 0.42 & 13.58 & 180 & -0.01 & 0.37 & 12.97 \\
46 & -0.04 & 0.52 & 12.92 & 110 & +0.82 & 0.52 & $14.83:$ & 186 & -0.13 & 0.37 & 12.58 \\
54 & -0.12 & 0.31 & 12.66 & 111 & +0.81 & 0.51 & 15.05 & 187 & +0.25 & 0.47 & 13.60 \\
56 & -0.10 & 0.35 & 13.19 & 119 & -0.02 & 0.42 & 13.65 & 190 & +0.86 & 0.51 & 14.81 \\
59 & +0.58 & 0.38 & 14.18 & 134 & -0.04 & 0.47 & 13.66 & 194 & -0.25 & 0.60 & 9.59 \\
60 & +0.24 & 0.48 & 13.52 & 144 & +0.58 & 0.54 & 14.27 & 195 & -0.25 & 0.43 & 9.41 \\
65 & -0.25 & 0.34 & 9.61 & 147 & -0.02 & 0.47 & 13.75 & 199 & -0.09 & 0.49 & 13.18 \\
68 & -0.11 & 0.32 & 12.89 & 149 & -0.06 & 0.44 & 12.41 & 203 & -0.01 & 0.53 & 13.66 \\
70 & $+0.50:$ & 0.38 & 13.34 & 150 & -0.09 & 0.39 & 12.21 & 207 & -0.07 & 0.65 & 13.25 \\
71 & -0.01 & 0.37 & 13.76 & 151 & -0.06 & 0.37 & 12.68 & 212 & +0.55 & 0.37 & 13.46 \\
73 & +0.73 & 0.49 & 14.17 & 152 & -0.22 & 0.41 & 11.23 & 213 & -0.20 & 0.37 & 10.85 \\
78 & +0.70 & 0.39 & 14.80 & 153 & $+0.89:$ & $0.36^{\mathrm{b}}$ & 15.45 & 214 & -0.24 & 0.39 & 9.89 \\
79 & +0.76 & 0.51 & 14.80 & 154 & +0.56 & 0.43 & 13.33 & 215 & -0.09 & 0.49 & 11.74 \\
88 & -0.13 & 0.31 & 12.41 & 157 & -0.11 & 0.50 & 13.18 & 218 & -0.08 & 0.37 & 12.61 \\
89 & -0.13 & 0.58 & 11.83 & 159 & 0.12 & 0.42 & 12.29 & 223 & +0.58 & 0.46 & 13.83 \\
92 & -0.26 & 0.32 & 9.32 & 161 & -0.24 & 0.40 & 9.97 & & & & \\
\hline \hline
\end{tabular}

Notes: (a) Reddening equivalent to that of a B0 star.

(b) Space reddening adopted from closely-adjacent stars. 


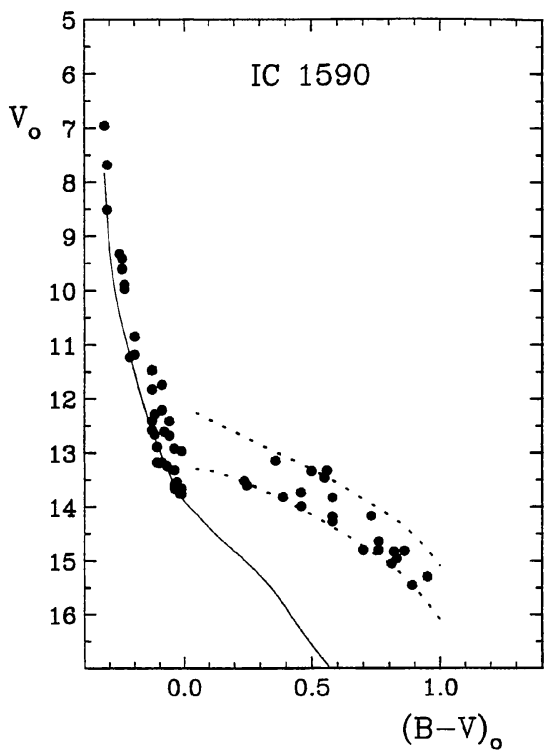

FIG. 10. Reddening-corrected color-magnitude diagram for likely members of IC 1590. The continuous line represents the ZAMS corresponding to a distance modulus of $V_{0}-M_{V}=12.34$, and dotted lines are isochrones corresponding to stellar ages of $10^{6}$ and $3.5 \times 10^{6}$ years (upper and lower, respectively).

ann 1970; Collins \& Smith 1985), then the cluster must be exactly $3.5 \times 10^{6}$ years old, with essentially no spread in age. The tight lower envelope evident for 9 of the pre-mainsequence stars in IC 1590 and their remarkably good fit to a $3.5 \times 10^{6}$ year isochrone (with an uncertainty of only \pm 0.2 $\times 10^{6}$ years) interpolated from the theoretical relations published by Palla \& Stahler (1993) provide a good case for the second interpretation. The remaining 13 pre-main-sequence stars $(59 \%)$ lie above the $3.5 \times 10^{6}$ year isochrone, but within the canonical 0.75 difference corresponding to an unresolved pair of equally-bright stars (or to light contamination from a circumstellar disk equal to that from the star). Their relative numbers are consistent with that expected for binary members of the cluster.

The gap in Fig. 10 which separates main-sequence stars of spectral type $B 9.5\left[(B-V)_{0}=-0.02\right]$ from the bluest premain-sequence members of spectral type A8/9 $\left[(B-V)_{0}=+0.24\right]$ appears to be real. There are no candidate cluster stars in the observed sample which fall within that color range, and our sample is complete for potential members in the appropriate magnitude interval. Ulrich (1971) previously explained such gaps as a consequence of the temporary halting of gravitational collapse by ${ }^{3} \mathrm{He}$ burning in the late stages of contraction of pre-main-sequence stars, followed by a relatively rapid collapse to the hydrogenburning main sequence. The effect disappears in the more recent models of Palla \& Stahler (1993) because of differences in the sizes of the convection zones for stars of comparable mass, so the gap must have another explanation. Such a feature is not observed in a similarly-derived colormagnitude diagram for NGC 2264 (Tumer, unpublished), a cluster of only slightly greater age than IC 1590 .

The redwards displacement of main-sequence B-type

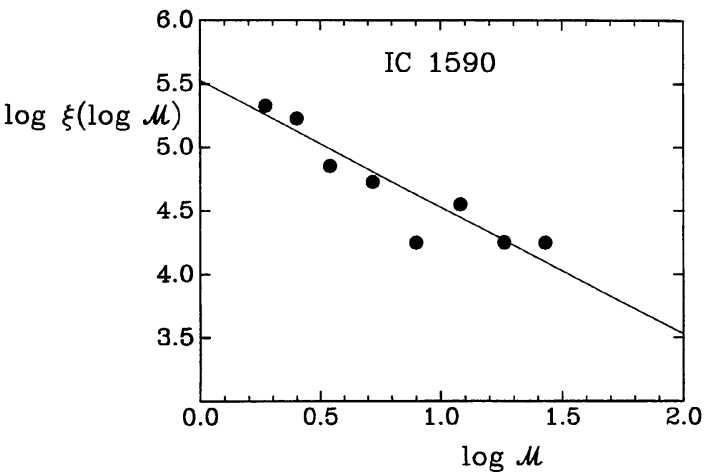

FIG. 11. The initial mass function for stars identified as likely members of IC 1590. Each point corresponds to the number of stars falling in specific magnitude intervals of $M_{V}$, namely (from right to left) $-5,-4 \ldots+2$. The straight line is the result of a statistical fit to the data, and has a slope $\Gamma=d \log \xi(\log \mathscr{M}) / d \log \mathscr{H}=-1.00 \pm 0.21$.

members of IC 1590 away from the ZAMS in Fig. 10 is not a result of evolutionary age spread. The spectroscopic observations indicate that rapid rotation is relatively common among main-sequence members of IC 1590, and that, in combination with binarity, could fully account for the observed scatter (Turner 1996b; Lastennet \& Valls-Gabaud 1996) as well as the apparent displacement of star 157 below the ZAMS (it must be a rapid rotator viewed pole-on) and the apparent presence of stars of $2.2 \mathscr{M}_{\odot}$ near the ZAMS (see previous comments). The intrinsic photometric scatter for cluster stars is so small that such effects are clearly apparent once the data have been corrected for the effects of differential interstellar reddening (cf. Turner 1996b).

Our photometric identification of 63 likely members of IC 1590 to a limiting absolute magnitude of $M_{V} \leqslant+3$ provides a good hold on the cluster luminosity function. The tie-in to the initial mass function for the cluster is less accurate, however, owing to possible systematic effects attributable to rapid rotation (see above) and the uncertain contribution of close companions, particularly among the pre-main-sequence stars. In Fig. 11 we have transformed counts for the number of stars per unit magnitude bin in absolute visual magnitude $M_{V}$ to the number of stars per square kiloparsec per logarithmic mass interval according to the precepts of Scalo (1986). HD 5005b has been included in the counts using its magnitude difference of $1 \mathrm{~m} .3$ relative to HD 5005a (Worley 1972), and the mass intervals for pre-main-sequence stars were established with reference to published evolutionary tracks. We also adjusted the counts for the latter to account for the likely multiplicity of most of the objects, and limited the analysis to $M_{V} \leqslant+2.5$, the realistic luminosity limit to which our survey can be considered relatively complete.

Least squares, regression and non-parametric techniques were used to fit straight lines to the data of Fig. 11, and the results were found to be relatively insensitive to the method of treatment. Uncertainties in the cluster distance modulus were also unimportant with regard to the distribution of data points. The inferred best-fitting slope for the initial mass function in IC 1590 is $\Gamma=d \log \xi(\log \mathscr{O}) / d \log \mathscr{M b}$ $=-1.00 \pm 0.21$. The cluster mass function is therefore char- 
acterized by a somewhat shallower slope than the value of $\Gamma=-1.35$ obtained by Salpeter (1955) for the "original mass function." However, the cluster population appears to be remarkably similar in its stellar mass distribution to other very young clusters and associations in the Galaxy, such as Cyg OB2 (Massey \& Thompson 1991), Tr 14 and Tr 16 (Massey \& Johnson 1993), NGC 6611 (Hillenbrand et al. 1993), the core region of IC 1805 (Ninkov et al. 1995), and NGC 6531 (Forbes 1996), for which the derived slopes for the initial mass function are $\Gamma=-1.0 \pm 0.3,-1.3 \pm 0.2$, $-1.1 \pm 0.3,-1.38 \pm 0.19$, and $-1.29 \pm 0.17$, respectively. From the data published by Joshi \& Sagar (1983) for the extended region of IC 1805, Ninkov et al. (1995) obtained a value of $\Gamma=-1.25 \pm 0.17$, which is marginally smaller than the slope derived for the core region.

\section{DISCUSSION}

Our original intent in studying IC 1590 was to remedy the previous neglect of the cluster in programs of photometric observation. Existing studies of the cluster field at microwave and radio wavelengths can now be tied to a much more complete optical picture of the cluster and its surroundings. NGC 281 is a relatively bright $\mathrm{H}$ II region, and the bright components of its central trapezium system HD 5005 are fairly hot main-sequence O-type stars that cannot be older than about $3.5 \times 10^{6}$ years. A cluster age of $\sim 3 \times 10^{6}$ years was estimated by Henning et al. (1994).

The mix of photoelectric and CCD $U B V$ photometry, MK spectroscopy, $K$-band and $I$-band photometry, and detailed star counts for the cluster field presented here provides all of the information necessary for a detailed analysis of the cluster and its member stars. One of our original concerns, the cluster extinction law, has been thoroughly investigated, and we find that the extinction law does appear to be somewhat anomalous in comparison with the properties exhibited by nearby dust (Turner 1994, 1996a). The derived value of $R_{V}=3.44 \pm 0.07$ is very well anchored in the $K$-band excesses, the $I$-band excesses, and a variable-extinction analysis of ZAMS stars, and appears to be associated with dust along the line of sight to IC 1590. The visual reddening relation of slope $\mathrm{E}_{U-B} / \mathrm{E}_{B-V}=0.735$ for the cluster is quite appropriate for this region of the galactic plane. Although there are a few elephant trunks and Bok globules visible against the emission of NGC 281 and an obvious incursion of foreground dust into the region from the south, there is otherwise little photometric evidence for the presence of interstellar dust within the cluster boundaries.

The distance to the cluster established by main-sequence fitting is $2.94 \pm 0.15 \mathrm{kpc}$, significantly larger than the estimate of $2.0 \mathrm{kpc}$ given by Sharpless (1954) and smaller than the value of $3.5 \mathrm{kpc}$ derived by Henning et al. (1994). At the distance found here, the main components of the HD 5005 trapezium system have projected separations of $0.02-0.13 \mathrm{pc}$ (4,000-26,000 A.U.). The cluster itself is about $12 \mathrm{pc}$ in diameter, somewhat smaller than the $17 \mathrm{pc}$ diameter of the $\mathrm{H}$ II region in which it is embedded. The large Bok globule located near cluster stars 59, 69, 93, and 99 measures about $0.8 \times 0.4 \mathrm{pc}$ in projected dimensions, while the smaller globule located between stars 215, 216 and 217 measures about $0.4 \times 0.2 \mathrm{pc}$.

The color-magnitude diagram of IC 1590 (Fig. 10) is a classic example of the H-R diagram for a very young cluster. The main sequence is populated from spectral types 06.5 to B9.5, and the small amount of scatter visible above the ZAMS is about what one expects from a combination of rapid rotation and binarity affecting the stellar colors and magnitudes (Turner 1996b; Lastennet \& Valls-Gabaud 1996). Rapid rotation for several cluster stars is, in fact, indicated by the spectroscopic observations and is inferred from the manner in which the lower main sequence is populated. Cluster pre-main-sequence stars are detected over a color interval that corresponds to spectral types ranging from A8/9 to G8. Their identification depends directly on the star count data and the derived field reddenings of nearby cluster main-sequence members. A comparison with the predictions from theoretical evolutionary models for contracting stars given by Palla \& Stahler (1993) indicates that the stars are entirely consistent with a cluster age of $3.5( \pm 0.2) \times 10^{6}$ years. A spread in age for cluster members also seems unlikely given that the main sequence of the cluster is relatively smoothly populated with stars down to masses of $2.6 \mathscr{M}_{\odot}$ or less. What scatter is evident for pre-main-sequence stars is most likely caused by binarity or contamination from circumstellar disks.

The pre-main-sequence stars detected in IC 1590 are also rather interesting by virtue of the fact that they show no evidence for significant variability. Since the sample lies very close to the regime for $\mathrm{T}$ Tauri variables and embedded protostars, a somewhat deeper survey than that presented here should be capable of detecting cluster members of that type. In that regard, the field of IC 1590 would make an excellent program object for the Hubble Space Telescope. The characteristics of cluster stars brighter than $V=17$ are well-established by this study, and much information related to the star formation process could be obtained by imaging the field to fainter magnitude limits. One such study using the facilities of the U.S. Naval Observatory is already in progress by one of us (Guetter \& Luginbuhl 1996).

This investigation was supported in part by funds awarded through the Natural Sciences and Engineering Research Council of Canada (NSERC) to D.G.T. The assistance of Norman Scrimger and a variety of research assistants in the early stages of this study, and of Michael Seymour in the last stages, is gratefully acknowledged by D.G.T.

\section{REFERENCES}

Abt, H. A. 1986, ApJ, 304, 688

Bessel, M. S. 1979, PASP, 91, 589

Collins, G. W., II, \& Smith, R. C. 1985, MNRAS, 213, 519
Conti, P. S., \& Alschuler, W. R. 1971, ApJ, 170, 325

Elmegreen, B. G., \& Lada, C. J. 1978, ApJ, 219, 467

Fernie, J. D. 1983, PASP, 95, 782 
FitzGerald, M. P. 1970, A\&A, 4, 234

Forbes, D. 1996, AJ, 112, 1073

Guetter, H. H. 1968, PASP, 80, 197

Guetter, H. H. 1979, AJ, 84, 1846

I' Guetter, H. H., \& Hewitt, A. A. 1984, PASP, 96, 441

II Guetter, H. H., \& Luginbuhl, C. B. 1996, BAAS, 28, 887

Guetter, H. H., \& Vrba, F. J. 1989, AJ, 98, 611

Guetter, H. H., Turner, D. G., \& Scrimger, J. N. 1985, BAAS, 17, 557

Henning, T., Martin, K., Reimann, H.-G., Launhardt, R., Leisawitz, D., \& Zinnecker, H. 1994, A\&A, 288, 282

Hiltner, W. A. 1956, ApJS, 2, 389

Hillenbrand, L. A., Massey, P., Strom, S. E., \& Merrill, K. M. 1993, AJ, 106, 1906

Israel, F. P. 1977, A\&A, 60, 233

Johnson, H. L. 1965, ApJ, 141, 923

Johnson, H. L. 1966, ARA\&A, 4, 193

Johnson, H. L. 1968, in Nebulae and Interstellar Matter, edited by B. M. Middlehurst and L. H. Aller (University of Chicago Press, Chicago), Chap. 5

Johnson, P. G., White, N. J., \& Pedlar, A. 1981, MNRAS, 196, 995

Joshi, U. C., \& Sagar, R. 1983, JRASC, 77, 40

Keenan, P, C. 1963, in Basic Astronomical Data, edited by K. Aa. Strand (University of Chicago Press, Chicago), Chap. 8

Kholopov, P. N. 1969, SvA, 12, 625

Lastennet, E., \& Valls-Gabaud, D. 1996, in The Origins, Evolution, and Destinies of Binary Stars in Clusters, ASP Conf. Ser. 90, edited by E. F. Milone and J.-C. Merrnilliod (ASP, San Francisco), p. 464

Leisawitz, D. 1988, NASA Ref. Publ. No. 1202

Leisawitz, D., Bash, F. N., \& Thaddeus, P. 1988, ApJS, 70, 731

Leonard, P. J. T. 1988, AJ, 95, 108

Maeder, A., \& Peytremann, E. 1970, A\&A, 7, 120
Massey, P., \& Johnson, J. 1993, AJ, 105, 980

Massey, P., \& Thompson, A. B. 1991, AJ, 101, 1408

McCuskey, S. W. 1965, in Galactic Structure, edited by A. Blaauw and M. Schmidt (University of Chicago Press, Chicago), Chap. 1

Mendoza, E. E. V. 1968, Publ. Univ. Chile Dept. Astron., Vol. I, No. 7,106

Morgan, W. W., Code, A. D., \& Whitford, A. E. 1955, ApJS, 2, 41

Ninkov, Z., Bretz, D. R., \& Easton, Jr., R. L. 1995, AJ, 110, 2242

Palla, F., \& Stahler, S. W. 1993, ApJ, 418, 414

Roger, R. S., \& Pedlar, A. 1981, A\&A, 94, 238

Sharpless, S. 1954, ApJ, 119, 334

Salpeter, E. E. 1955, ApJ, 121, 161

Scalo, J. M. 1986, Fundam. Cosmic Phys., 11, 1

Stetson, P. B. 1987, PASP, 99, 191

Turner, D. G. 1976a, AJ, 81, 97

Turner, D. G. 1976b, AJ, 81, 1125

Turner, D. G. 1979, PASP, 91, 642

Turner, D. G. 1980, ApJ, 240, 137

Turner, D. G. 1989, AJ, 98, 2300

Turner, D. G. 1992, AJ, 104, 1865

Turner, D. G. 1994, RMxA\&A, 29, 163

Turner, D. G. 1996a, AJ, 111, 828

Turner, D. G. 1996b, in The Origins, Evolution, and Destinies of Binary Stars in Clusters, ASP Conf. Ser. 90, edited by E. F. Milone and J.-C. Merrnilliod (ASP, San Francisco), p. 443

Turner, D. G., Forbes, D., \& Pedreros, M. 1992, AJ, 104, 1132

Turner, D. G., Garrison, R. F., \& Morris, S. C. 1994, JRASC, 88, 303 Ulrich, R. K. 1971, ApJ, 168, 57

van den Bergh, S., \& Sher, D. 1960, Publ. DDO, 2, 201

Walborn, N. R. 1973, AJ, 78, 1067

Worley, C. E. 1972, Publ. USNO, Vol. XXII, Part IV

Yoss, K. M. 1961, ApJ, 134, 809 